November 2004 • NREL/TP-500-34421

\title{
Extrapolation of Extreme and Fatigue Loads Using Probabilistic Methods
}

P.J. Moriarty, W.E. Holley, and S.P. Butterfield

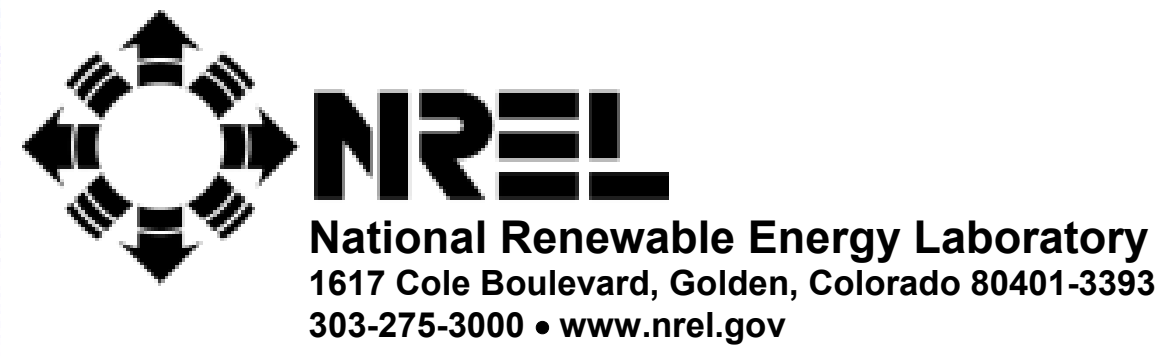

Operated for the U.S. Department of Energy

Office of Energy Efficiency and Renewable Energy

by Midwest Research Institute • Battelle

Contract No. DE-AC36-99-GO10337 


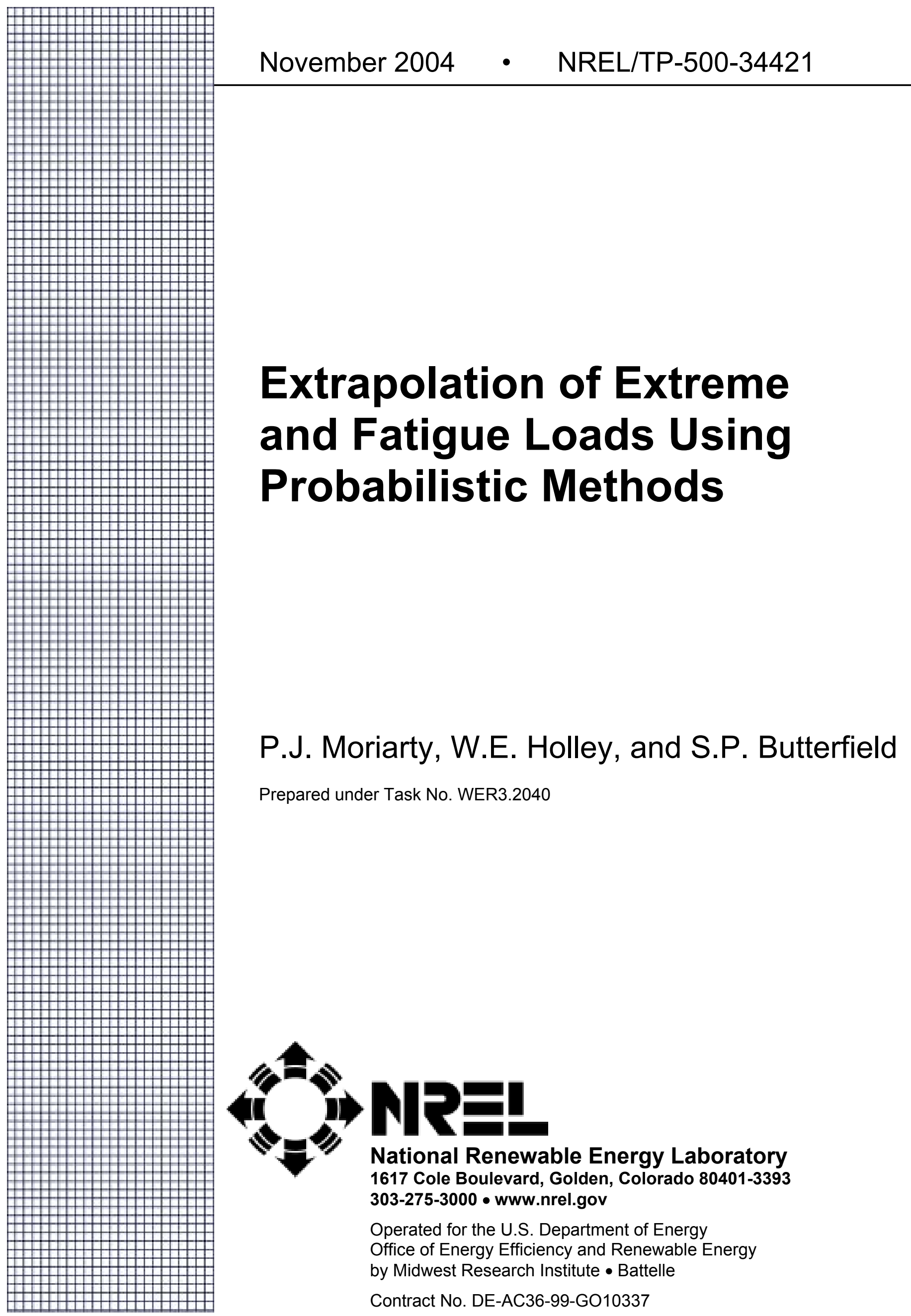




\section{NOTICE}

This report was prepared as an account of work sponsored by an agency of the United States government. Neither the United States government nor any agency thereof, nor any of their employees, makes any warranty, express or implied, or assumes any legal liability or responsibility for the accuracy, completeness, or usefulness of any information, apparatus, product, or process disclosed, or represents that its use would not infringe privately owned rights. Reference herein to any specific commercial product, process, or service by trade name, trademark, manufacturer, or otherwise does not necessarily constitute or imply its endorsement, recommendation, or favoring by the United States government or any agency thereof. The views and opinions of authors expressed herein do not necessarily state or reflect those of the United States government or any agency thereof.

Available electronically at http://www.osti.gov/bridge

Available for a processing fee to U.S. Department of Energy and its contractors, in paper, from:

U.S. Department of Energy

Office of Scientific and Technical Information

P.O. Box 62

Oak Ridge, TN 37831-0062

phone: 865.576 .8401

fax: 865.576 .5728

email: mailto:reports@adonis.osti.gov

Available for sale to the public, in paper, from:

U.S. Department of Commerce

National Technical Information Service

5285 Port Royal Road

Springfield, VA 22161

phone: 800.553 .6847

fax: 703.605.6900

email: orders@ntis.fedworld.gov

online ordering: http://www.ntis.gov/ordering.htm 


\section{Abstract}

In this study, researchers used probabilistic methods to determine the long-term extreme and fatigue loads of two different $1.5 \mathrm{MW}$ wind turbine designs. The method of moments was used to calculate short-term distributions of data for varying wind conditions. The data of interest for the study was limited to the in-plane and out-of-plane bending loads at the roots of the blades of both turbines.

We examined four different distributions for fitting of the short-term data. It was discovered that thresholding the data before fitting the short-term distribution increased the accuracy of the fitted distribution in following the trends of the largest extreme and fatigue cycle values for a given wind condition. The optimal threshold value for extreme loads was found to be the mean value plus 1.4 times the standard deviation of the original time series of the bending load. For fatigue loading, the optimal threshold value was the 99th percentile value of the original cyclic distribution. Rainflow counted cyclic amplitudes from time series simulations were also adjusted using a Goodman correction to account for nonzero cyclic midpoint values.

When fitting a short-term distribution to a given data set, the higher-order moments were found to have the greatest amount of uncertainty and also the largest influence on the extrapolated long-term loads. Using large data sets, smoothing of the statistical moments between wind conditions and parametrically modeling moments of the distribution reduced this uncertainty.

The short-term distributions were integrated over all wind conditions along with the joint probability distribution function of the mean wind speed and turbulence level to produce the longterm distributions. From the long-term distributions of extremes, the 1- and 50-year extreme loads were estimated. From the long-term distributions of fatigue cycles, the fatigue lifetime of the wind turbine could be estimated, which was found to be highly dependent on the material fatigue exponent. Long-term distributions of fatigue cycles showed that out-of-plane blade bending loads produced the largest amplitude cycles. Hence, these bending loads were used to estimate the lifetime of both turbines under the assumption of fixed ultimate bending strength. Lifetimes were not found to decrease significantly when accounting for the tail of the fitted distribution for the large data set used.

We examined several alternative methods to the full integration technique for calculating longterm distributions. One alternative required extrapolating the turbulence level at each mean wind speed before simulation. This method greatly reduced the number of simulations required, but was found to be sensitive to the cut-out wind speed for stall regulated turbines. Another alternative was to use a parametric model of the statistical moments of the data sets. The parametric model predicted nearly identical loads to the full integration technique and required less data. Another simplified method for calculating extremes and lifetimes using a deterministic, elevated turbulence intensity produced slightly conservative results relative to the full distribution method and significantly reduced the number of simulations required.

The accuracy of the extrapolation techniques was quantified by comparing the results to a oneyear direct simulation of turbine operation. For extreme values, the extrapolation technique accurately predicted the long-term distributions of the in-plane bending loads for both turbines, and the out-of-plane bending load for the stall-regulated turbine, but overpredicted the load distributions for the out-of-plane bending load on the pitch-regulated turbine. For fatigue cycles, the extrapolation technique accurately predicted the long-term distributions of the in-plane bending loads and overpredicted the out-of-plane bending loads for both turbines. Regardless of errone- 
ous predictions of bending load distributions, lifetime estimates using extrapolation were on the order of estimates from the one-year simulation. 


\section{Table of Contents}

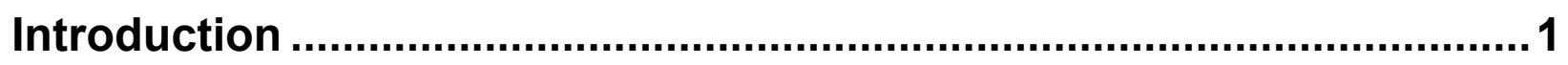

Wind Environment ........................................................................... 2

Simulation Data ....................................................................................... 3

Method of Moments ............................................................................ 4

Extreme Value Analysis...................................................................... 7

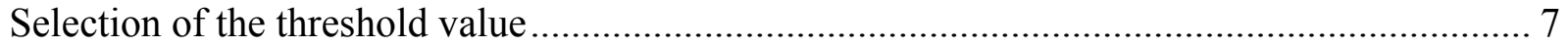

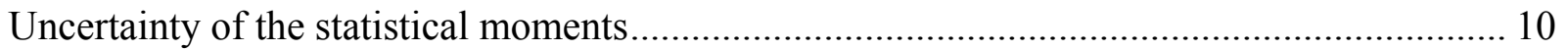

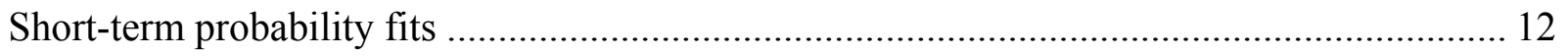

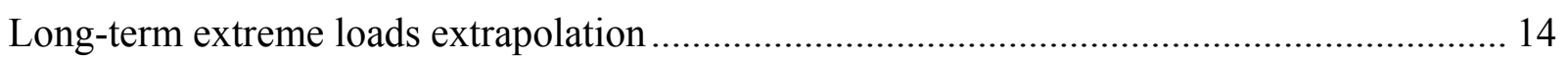

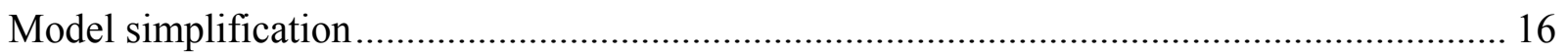

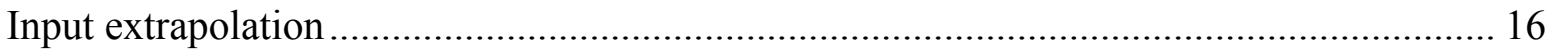

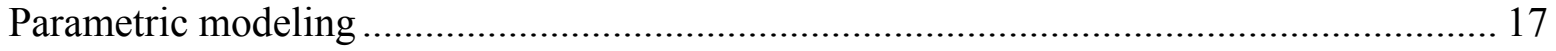

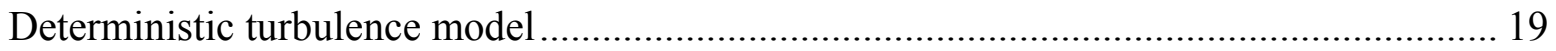

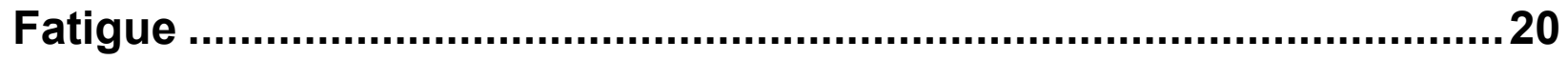

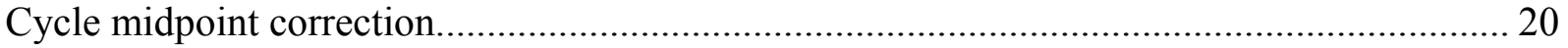

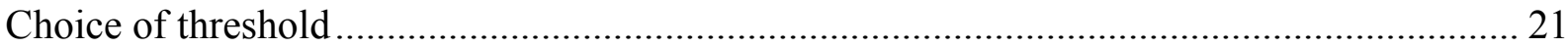

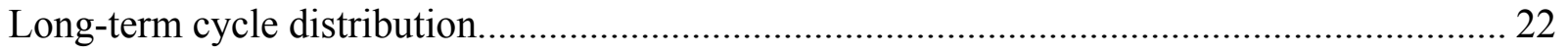

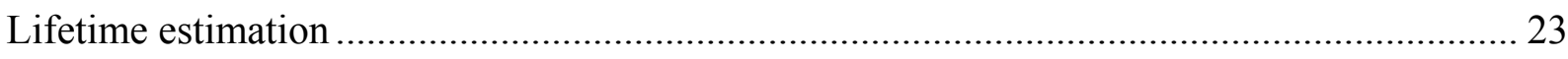

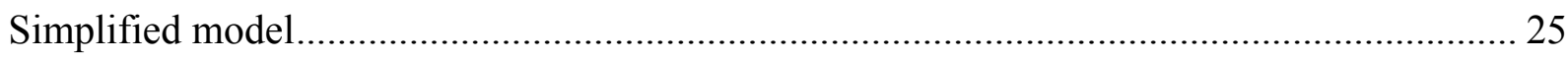

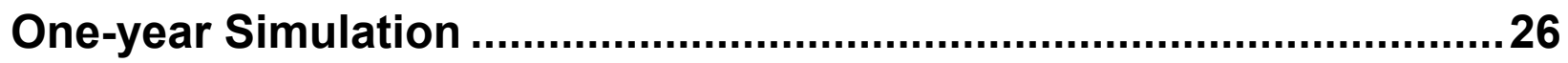

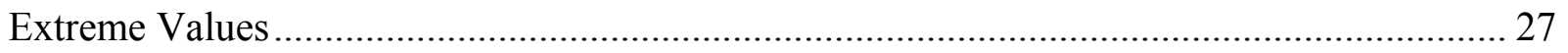

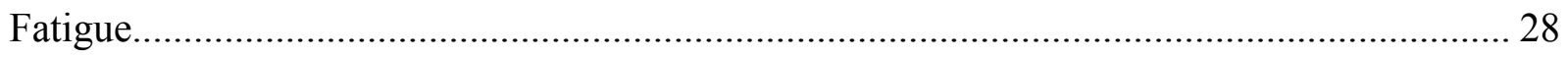

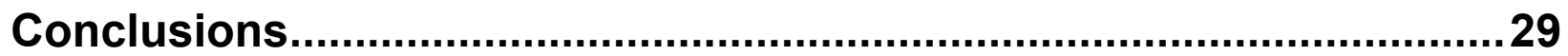

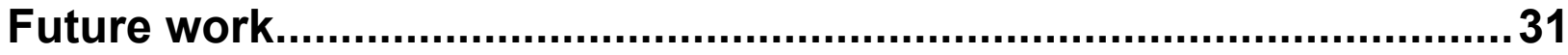

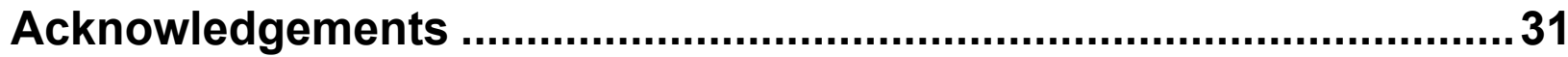

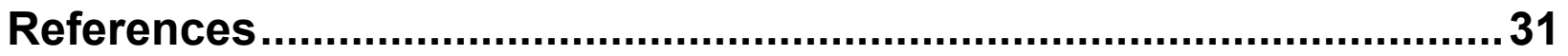




\section{Table of Figures}

Figure 1. Joint probability density function of mean wind speed and turbulence level............. 3

Figure 2. Contour plot of joint probability density function .................................................. 3

Figure 3. Effect of threshold on accuracy of fit for extreme values (Out-of-plane bending load of pitch-regulated turbine)

Figure 4. Normalized probability distributions of extreme values (Out-of-plane bending load of pitch-regulated turbine)

Figure 5. Calculated bending load threshold vs. number of aggregate 10-minute simulations (Out-of-plane bending load of pitch-regulated turbine)...... 10

Figure 6. Calculated shifted mean value of maxima vs. number of aggregate 10-minute simulations (Out-of-plane bending load of pitch-regulated turbine)

Figure 7. Calculated standard deviation of maxima vs. number of aggregate 10-minute simulations (Out-of-plane bending load of pitch-regulated turbine)

Figure 8. Calculated skewness of maxima vs. number of aggregate 10-minute simulations (Out-of-plane bending load of pitch-regulated turbine).

Figure 9. Calculated number of load maxima vs. number of aggregate 10-minute simulations (Out-of-plane bending load of pitch-regulated turbine)...

Figure 10. Skewness of load maxima for pitch-regulated turbine (Out-of-plane bending load) . 12

Figure 11. Skewness of load maxima for stall-regulated turbine (Out-of-plane bending load)... 12

Figure 12. Effect of moment smoothing on long-term probability distribution for pitch-regulated turbine (Out-of-plane bending load)

Figure 13. Probability of exceedance, Vmean $=10 \mathrm{~m} / \mathrm{s}, \quad=0.2 \mathrm{~m} / \mathrm{s}$, pitch-regulated turbine (Out-of-plane bending load)

Figure 14. Probability of exceedance, Vmean $=10 \mathrm{~m} / \mathrm{s}, \quad=1.8 \mathrm{~m} / \mathrm{s}$, pitch-regulated turbine (Out-of-plane bending load)

Figure 15. Probability of exceedance, Vmean $=10 \mathrm{~m} / \mathrm{s}, \quad=5.0 \mathrm{~m} / \mathrm{s}$, pitch-regulated turbine (Out-of-plane bending load)

Figure 16. Probability of exceedance, Vmean $=10 \mathrm{~m} / \mathrm{s}, \quad=5.0 \mathrm{~m} / \mathrm{s}$, stall-regulated turbine (Out-of-plane bending load)

Figure 17. Long-term exceedance probability distributions for out-of-plane load of the pitch-regulated turbine.

Figure 18. Long-term exceedance probability distributions for out-of-plane load of the stall-regulated turbine

Figure 19. Comparison of effect of turbulence distribution on long-term exceedance probability for out-of-plane loads of the pitch-regulated turbine 15

Figure 20. Exceedance probability for turbulence levels at Vmean $=10 \mathrm{~m} / \mathrm{s}$. 16 
Figure 21. Extreme value in $10 \mathrm{~min}$. simulation using extrapolated 50-year turbulence level (Out-of-plane bending load of stall-regulated turbine).

Figure 22. Load threshold value compared to parametric data fit (Out-of-plane bending load of pitch-regulated turbine).

Figure 23. Shifted mean value of load maxima compared to parametric data fit (Out-of-plane bending load of pitch-regulated turbine).

Figure 24. Standard deviation of load maxima compared to parametric data fit (Out-of-plane bending load of pitch-regulated turbine).

Figure 25. Number of load maxima compared to parametric data fit (Out-of-plane bending load of pitch-regulated turbine) 18

Figure 26. Long-term probability distributions from empirical vs. parametric models for a pitchregulated turbine 19

Figure 27. Long-term probability distributions from empirical vs. parametric models for a stallregulated turbine .....

Figure 28. Comparison of predicted extreme loads for a pitch-regulated turbine using different turbulence distributions.

Figure 29. Comparison of predicted extreme loads for a stall-regulated turbine using different turbulence distributions.

Figure 30. Threshold effect on exceedance probability and fitted distribution for out-of-plane bending load on the pitch regulated turbine.

Figure 31. Normalized probabilities of exceedance for out-of-plane bending load on the pitch regulated turbine. 22

Figure 32. Expected number of cycles per year for pitch-regulated turbine. 23

Figure 33. Expected number of cycles per year for stall-regulated turbine. ............................ 23

Figure 34. Estimated fatigue life for pitch-regulated turbine............................................... 25

Figure 35. Estimated fatigue life for stall-regulated turbine. .................................................. 25

Figure 36. Estimated fatigue life for pitch-regulated turbine using deterministic turbulence level 26

Figure 37. Estimated fatigue life for stall-regulated turbine using deterministic turbulence level. 26

Figure 38. Total number of 10-minute simulations per wind condition in one-year ................ 27

Figure 39. Contour of number of 10-minute simulations per wind condition and joint probability density function

Figure 40. Predicted long-term distributions of extreme loads compared to direct simulation data for pitch-regulated turbine. 28

Figure 41. Predicted long-term distributions of extreme loads compared to direct simulation data for stall-regulated turbine. 28

Figure 42. Predicted long-term distributions of fatigue loads compared to direct simulation data for pitch-regulated turbine 
Figure 43. Predicted long-term distributions of fatigue loads compared to direct simulation

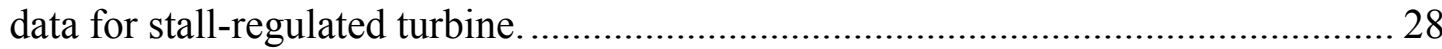

Figure 44. Predicted lifetimes compared to direct simulation data for pitch-regulated turbine... 29

Figure 45. Predicted lifetimes compared to direct simulation data for stall-regulated turbine.... 29 


\section{Introduction}

Over the past decade, researchers and industrial designers have become increasingly interested in probabilistic methods for wind turbine design. This interest has largely been driven by the desire to replace outdated empirical-based design models with more physically relevant models that can be accurately applied to the next generation of wind turbine designs. Probabilistic methods are well suited to this task because they use limited amounts of either experimental or simulation data from actual turbine designs to predict long-term loading. These methods can be used to extrapolate extreme and fatigue loads, as well as other quantities such as deflections, but this report will concentrate primarily on loads.

As far as predicting extreme loads, current design standards for wind turbines, governed by the IEC [1], require an estimate of the extreme 1-year and 50-year operating load. In the current version of the standard, these loads are specified as discrete events based on design experience and empirical models of older turbine designs. The standards also require using dynamic analysis of the fatigue loads to formulate the design loads for the turbine. Within the last few years, some concern has been raised that these design load cases are inaccurate and may be non-conservative for the next generation of turbines. In response to this concern, researchers have performed many studies using probabilistic methods to increase the accuracy of the predicted loads. In one of the earlier works, Veers and Winterstein [2] studied the method of moments for predicting long-term fatigue loading and also introduced a non-linear parametric model which was useful for extrapolating from limited data sets. A few years later, Ronold [3] also examined moment-based fatigue modeling, and studied the calibration of partial safety factors. A follow-up study by Ronold and Larsen [4], as well as Madsen et al. [5], showed that these techniques could be used for extreme load extrapolation and suggested that the statistics of the extremes follows a Gumbel distribution. Thomsen [6] used similar techniques and highlighted the importance of the cyclic midpoint load and turbulence intensity on fatigue extrapolation. Fitzwater and Winterstein [7] examined the effect of statistical uncertainty dependent on the type of data used in these extrapolation methods. Manuel et al. [8] continued the work of Veers and Winterstein using probabilistic methods and parametric models based on variations in mean wind speed and turbulence intensity. They also performed a detailed uncertainty analysis. Finally, driven by the complex nature of the moment-based calculations and expansive data sets often required, both Fitzwater et al. [9] and Moriarty et al. [10] examined several different simplifications for these techniques with varying degrees of success.

This study extends these previous works by examining moment-based probability methods, as well as the effects of using a joint distribution of mean wind speed and turbulence level and the statistical uncertainty of moment-based methods. This study will also introduce refinements such as compensating for non-zero cyclic midpoints in fatigue calculations, applying a threshold technique, and fully integrating all probable operating conditions. Simplifications of the method and parametric modeling are found to be useful for design without a significant loss in accuracy. Several methods of extrapolation are compared in the hopes of finding a consistent and accurate method that could be used by designers and written into a new version of the design standards. The results from extrapolation will also be compared to a direct 1-year simulation, which will give a measure of the accuracy of these techniques. 


\section{Wind Environment}

The driving force acting on wind turbines is, of course, the wind, which is traditionally modeled using two parameters: the mean wind speed and the standard deviation of the wind speed or turbulence level. Often, the turbulence level is given as a deterministic value conditional on mean wind speed. This is the case for the international design standard, IEC 61400-1 Ed. 2 [1], which assumes that turbulence level varies linearly with mean wind speed. However, measurements [11] have shown that turbulence level is not deterministic for a given mean wind speed, but is instead a random variable for both on-shore and off-shore sites. In order to account for this variability, along with the variation of mean wind speed a joint probability density function for both turbulence level and wind speed is proposed. This model assumes that mean wind speed follows a Rayleigh distribution and turbulence level is distributed like a log-normal function. The joint density function is described by

$$
f_{V, T}(v, t)=f_{T \mid V}(t \mid v) f(v)
$$

where the marginal mean wind speed distribution is defined by

$$
\begin{aligned}
& f_{V}(v)=\frac{2 v}{\alpha^{2}} \exp \left[-\left(\frac{v}{\alpha}\right)^{2}\right] \\
& \alpha=\frac{2 \mu_{V}}{\sqrt{\pi}}
\end{aligned}
$$

and the log-normal conditional turbulence distribution is

$$
\begin{aligned}
& f_{T \mid V}(t \mid v)=\frac{1}{\sqrt{2 \pi} \zeta t} \exp \left[-\frac{1}{2}\left(\frac{\ln (t)-\lambda}{\zeta}\right)^{2}\right] \\
& \zeta=\sqrt{\ln \left(\delta_{T \mid V}^{2}+1\right)} \\
& \lambda=\ln \left(\mu_{T \mid V}\right)-\frac{1}{2} \zeta^{2} \\
& \delta_{T \mid V}=\frac{\sigma_{T \mid V}}{\mu_{T \mid V}} \\
& \mu_{T \mid V}=I_{r e f}(0.75 v+c) \\
& \sigma_{T \mid V}=1.44 I_{r e f}
\end{aligned}
$$

where $\mu_{V}=10 \mathrm{~m} / \mathrm{s}, I_{r e f}=0.16$, and $c=3.8$ for an IEC class I-A wind site [1]. Note that the $I_{r e f}$ parameter has been defined to represent the mean value rather that the mean plus one standard deviation as given in IEC 61400-1 Ed.2. Figures 1 and 2 show the joint probability density as a function of mean wind speed and turbulence level (sigma). 


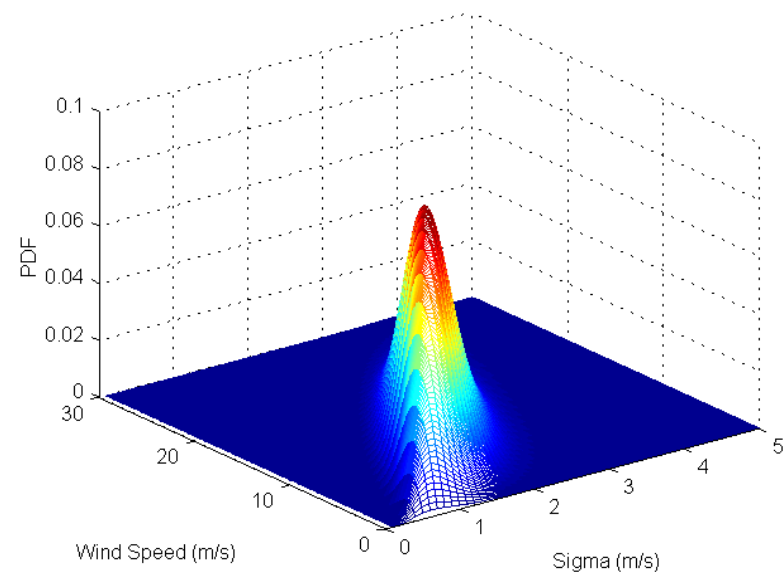

Figure 1. Joint probability density function of mean wind speed and turbulence level

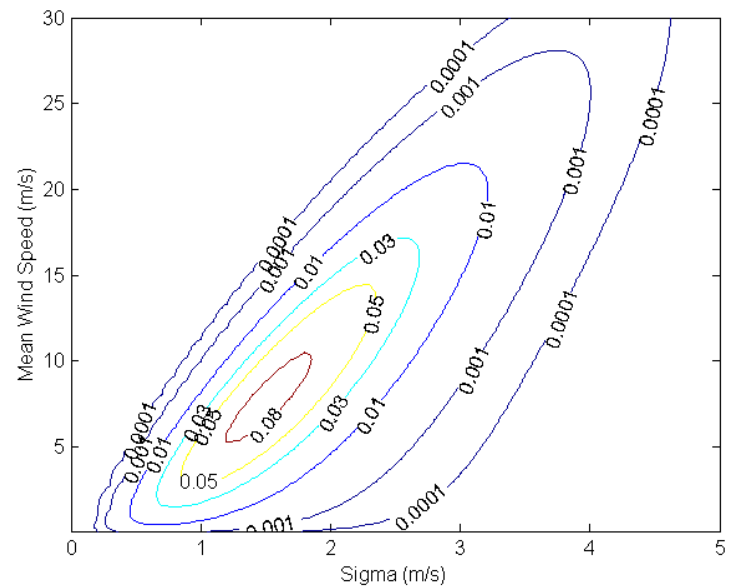

Figure 2. Contour plot of joint probability density function

Because of the complexity of this distribution, a more straightforward normal distribution of turbulence level was also examined. The equation for the resulting conditional turbulence distribution is given by

$$
f_{T \mid V}(t \mid v)=\frac{1}{\sqrt{2 \pi} \sigma_{T \mid V}} \exp \left[-\frac{1}{2}\left(\frac{t-\mu_{T \mid V}}{\sigma_{T \mid V}}\right)^{2}\right]
$$

Comparisons between extrapolations using the normal and the log-normal distribution are made below.

\section{Simulation Data}

In this study, we examined two different wind turbine configurations; one that was stall regulated at higher wind speeds and one that was pitch regulated. Each turbine model consisted of a threebladed upwind rotor with twisted and tapered blades and a radius of $35 \mathrm{~m}$. While actually fictitious, the turbines represented the generic characteristics of typical turbines with a rated power of 1.5 MW. The stall-regulated turbine was assumed to run at a constant speed of 16.9 RPM while the pitch-regulated, variable speed machine had a rated speed of 20.4 RPM. The rated wind speeds of the pitch-regulated and stall-regulated turbines were $11 \mathrm{~m} / \mathrm{s}$ and $14 \mathrm{~m} / \mathrm{s} \mathrm{respectively.}$ The operating wind speed ranges of both turbines were between 5 and $25 \mathrm{~m} / \mathrm{s}$ in conditions specified for the IEC class I-A [1]. For the purpose of simplification, the turbines were assumed to operate $100 \%$ of the time between cut-in and cut-out wind speeds with $100 \%$ availability. The hub height for both rotors was $84 \mathrm{~m}$.

All simulations in this study were performed using SNWind [12] to generate 10-minute threedimensional turbulent wind files and the FAST aeroelastic code [13] to calculate the turbine response in these winds. The turbulent spectrum of each wind simulation was determined using the Kaimal spectral model specified in the IEC standard. The vertical wind shear was modeled using a power law exponent of 0.2 , also specified in the standard. The wind files were calculated using a $10 \times 10$ grid over the rotor plane and the same wind inflow conditions were used for both turbine models. In the FAST calculations, three blade modes (two flap and one edge) were modeled along with four tower modes (two in each direction). 
In order to examine each turbine's response in all probable wind conditions, a large data set of wind simulations was compiled. Wind simulations were run over a range of mean wind speeds between 5 and $25 \mathrm{~m} / \mathrm{s}$ (in $1 \mathrm{~m} / \mathrm{s}$ increments) and longitudinal turbulence levels between 0.2 and 5 $\mathrm{m} / \mathrm{s}$ (in $0.2 \mathrm{~m} / \mathrm{s}$ increments). Nine simulations per wind condition were calculated resulting in a total of 4725 simulations for each turbine. Each wind simulation used a different seed number to ensure statistical independence between simulations. In order to eliminate start up and numerical transients in each simulation, an additional initialization period of 50 seconds was also calculated and removed from each simulation prior to analysis. For low wind speeds of $7 \mathrm{~m} / \mathrm{s}$ or less, the stall-regulated machine operated at very high tip speed ratios, which caused instabilities in the dynamic wake model used in FAST. Therefore, for wind speeds of $7 \mathrm{~m} / \mathrm{s}$ and lower, the equilibrium wake model was used instead. The outputs considered from the simulations were the inplane and out-of-plane bending loads at the root of a blade. The local extreme values from each 10-minute simulation were extracted using a peak over threshold method, as described below. We calculated fatigue cycle amplitudes and midpoint values using a rainflow counting algorithm [14] on the concatenation of all nine files at each wind condition.

A modified version of the FITS code [15], a moment-based probability distribution fitting code, was used to calculate the short-term fitted probability distributions. Four different distributions were examined for calculating the short-term distributions: Gumbel, Weibull (which required knowledge of the mean and standard deviation of the distribution), a quadratic Weibull, and a 3parameter Weibull model (which required knowledge of the mean, standard deviation, and skewness of the distribution, as explained in the next section).

\section{Method of Moments}

The short-term probability distributions of extreme and fatigue loading were calculated using the method of statistical moments, where the cumulative probability distribution for each short-term data set was represented by an analytic function. This function was then used to determine the long-term total probability distribution by integration over all environmental wind conditions, as described below. Since the design requires a low total exceedance probability, this integration results in an evaluation of the short-term cumulative distribution function (CDF) over a range that will extend beyond the original data. Thus, it is important for the analytic representation to accurately represent the data and its trend for the largest values. Two analytic distributions were used in this study to describe the short-term CDFs. They were:

$$
\begin{aligned}
& \text { Gumbel: } F(x)=e^{-e^{-\left(\frac{x-x_{0}}{c}\right)}} \\
& \text { Weibull: } F(x)=1-e^{-\left(\frac{x-x_{0}}{c}\right)^{\alpha}}
\end{aligned}
$$

The Weibull distribution applies for values of $\mathrm{x}>\mathrm{x}_{0}$, whereas the Gumbel distribution applies for the full range of $\mathrm{x}$. In order to estimate the parameters in these models, a statistical momentbased method was used [15]. In this method, the statistical moments of the data are computed and then the parameters in the model are determined by matching analytical expressions for the moments in terms of the parameters. Given $n$ parameters in the model, the first $n$ statistical moments are used. 
It is convenient to express the distributions in terms of a normalized variable $y$, which is defined by a transformation of the variable $x$. The normalized statistical moments are given in Table 1 for these distributions. In cases where a linear transformation between $x$ and $y$ is used (i.e. $x=x_{0}+c y$ ), the normalized moments are given by

$$
\begin{aligned}
& \mu_{x}=x_{0}+c \mu_{y} \\
& \sigma_{x}=c \sigma_{y} \\
& \varepsilon_{x}=\varepsilon_{y}
\end{aligned}
$$

In this study, four different types of distribution fits were compared. The first (Gumbel) utilizes the standard Gumbel distribution, which has two parameters. In this case, the first two moments are used to estimate the parameters $x_{0}$ and c. Since $x$ is a linear transformation of $y$, this results in

$$
\begin{aligned}
& \hat{c}=\frac{\sigma_{x} \sqrt{6}}{\pi} \\
& \hat{x}_{0}=\mu_{x}-\hat{c} \gamma
\end{aligned}
$$

were $\gamma$ is Euler's constant given in Table 1.

The second method (Weibull) assumes $x_{0}$ is known a priori. In this case, there are also two unknown parameters, $\alpha$ and $c$. The coefficient of variation (COV) for the shifted data is defined by the ratio

$$
C O V=\frac{\sigma_{x}}{\mu_{x}-x_{0}}=\frac{\sigma_{y}}{\mu_{y}}=\sqrt{\frac{2 \alpha \Gamma\left(\frac{2}{\alpha}\right)-\Gamma\left(\frac{1}{\alpha}\right)^{2}}{\Gamma\left(\frac{1}{\alpha}\right)^{2}}}
$$

This equation is solved for the parameter $\hat{\alpha}$, which in turn gives

$$
\hat{c}=\frac{\mu_{x}-x_{0}}{\mu_{y}}=\frac{\hat{\alpha}\left(\mu_{x}-x_{0}\right)}{\Gamma\left(\frac{1}{\hat{\alpha}}\right)}
$$

where $\Gamma$ is the classical gamma function [16].

The third method (Quadratic Weibull) has three unknown parameters and assumes $y$ is related to $x$ by a quadratic distortion of the linear transformation for a standard Weibull fit. This distortion is adjusted to match the skewness in the data. Two forms are used depending on whether the skewness should be increased or decreased relative to the skewness in the data. In particular, defining

$$
u=\frac{x-x_{0}}{c}
$$

gives:

$$
\mathrm{y}=\mathrm{u}+\varepsilon \mathrm{u}^{2}
$$

for the case when the standard Weibull skewness is greater than the data, or 


$$
y=\frac{\sqrt{1+4 \varepsilon u}}{2 \varepsilon}
$$

for the case when the standard Weibull skewness is less than the data. From these relations, $u$ will be given in terms of a function, $f(y, \varepsilon)$ which is monotonically increasing with $y$ for positive $\varepsilon$. The statistical moments for $\mathrm{u}$ are then given by integrals that only depend on the two parameters $\varepsilon$ and $\alpha$. Thus,

$$
\begin{aligned}
& \mu_{u}(\varepsilon, \alpha)=\int_{0}^{\infty} f\left(z^{\frac{1}{\alpha}}, \varepsilon\right) k^{-z} d z \\
& \sigma_{u}(\varepsilon, \alpha)^{2}=\int_{0}^{\infty}\left(f\left(z^{\frac{1}{\alpha}}, \varepsilon\right)-\mu_{u}\right)^{2} e^{-z} d z \\
& \eta_{u}(\varepsilon, \alpha)=\int_{0}^{\infty}\left(\frac{f\left(z^{\frac{1}{\alpha}}, \varepsilon\right)-\mu_{u}}{\sigma_{u}}\right)^{3} e^{-z} d z
\end{aligned}
$$

In this method, the Weibull exponent, $\hat{\alpha}$, is determined from the COV as in the standard Weibull method and therefore depends on the assumed a priori shift in the data. The quadratic distortion parameter, $\hat{\varepsilon}$, is determined by numerical integration of the equations for the statistical moments and by matching the skewness parameter, $\eta_{u}$, to the skewness in the data. This results in the following equations for the parameters

$$
\begin{aligned}
& \eta_{u}(\hat{\varepsilon}, \hat{\alpha})=\eta_{x} \\
& \hat{c}=\frac{\sigma_{x}}{\sigma_{u}(\hat{\varepsilon}, \hat{\alpha})} \\
& \hat{x}_{0}=\mu_{x}-\hat{c} \mu_{u}(\hat{\varepsilon}, \hat{\alpha})
\end{aligned}
$$

The last method (Three Parameter Weibull) also has three unknown parameters and is able to match three statistical moments. It utilizes a linear transformation of the Weibull variable, but determines the Weibull exponent from the skewness, which is not dependent on a priori knowledge of the shift. Thus,

$$
\begin{aligned}
& \eta_{y}(\hat{\alpha})=\eta_{x} \\
& \hat{c}=\frac{\sigma_{x}}{\sigma_{y}(\hat{\alpha})} \\
& \hat{x}_{0}=\mu_{x}-\hat{c} \mu_{y}(\hat{\alpha})
\end{aligned}
$$

Because the first equation is transcendental in terms of the gamma functions given in Table 1, it must be solved iteratively. 


\begin{tabular}{|r|c|c|}
\hline & Weibull & Gumbel \\
\hline Cumulative distribution function, $\mathrm{F}(\mathrm{y})$ & $1-\mathrm{e}^{-\mathrm{y}^{\alpha}}$ & $\mathrm{e}^{-\mathrm{e}^{-\mathrm{y}}}$ \\
\hline Density function, $\mathrm{f}(\mathrm{y})=\mathrm{F}^{\prime}(\mathrm{y})$ & $\alpha \mathrm{y}^{\alpha-1} \mathrm{e}^{-\mathrm{y}^{\alpha}}$ & $\mathrm{e}^{-\left(\mathrm{y}+\mathrm{e}^{-\mathrm{y}}\right)}$ \\
\hline Mean, $\mu_{\mathrm{y}}=\int_{-\infty}^{+\infty} \mathrm{yf}(\mathrm{y}) \mathrm{dy}$ & $\frac{1}{\alpha} \Gamma\left(\frac{1}{\alpha}\right)$ & $\gamma \approx 0.577216$ \\
\hline Variance, $\sigma_{\mathrm{y}}{ }^{2}=\int_{-\infty}^{+\infty}\left(\mathrm{y}-\mu_{\mathrm{y}}\right)^{2} \mathrm{f}(\mathrm{y}) \mathrm{dy}$ & $\frac{2}{\alpha} \Gamma\left(\frac{2}{\alpha}\right)-\left(\frac{1}{\alpha} \Gamma\left(\frac{1}{\alpha}\right)\right)^{2}$ & $\frac{\pi^{2}}{6}$ \\
\hline Skewness, $\eta_{\mathrm{y}}=\int_{-\infty}^{+\infty}\left(\frac{\mathrm{y}-\mu_{\mathrm{y}}}{\sigma_{\mathrm{y}}}\right)^{3} \mathrm{f}(\mathrm{y}) \mathrm{dy}$ & $\frac{\frac{3}{\alpha} \Gamma\left(\frac{3}{\alpha}\right)-3\left(\frac{2}{\alpha} \Gamma\left(\frac{2}{\alpha}\right)\left(\frac{1}{\alpha} \Gamma\left(\frac{1}{\alpha}\right)\right)+2\left(\frac{1}{\alpha} \Gamma\left(\frac{1}{\alpha}\right)\right)^{3}\right.}{\left(\frac{2}{\alpha} \Gamma\left(\frac{2}{\alpha}\right)-\left(\frac{1}{\alpha} \Gamma\left(\frac{1}{\alpha}\right)\right)^{2}\right)^{\frac{3}{2}}}$ & $\frac{-\psi^{\prime \prime}(1)}{\left(\frac{\pi^{2}}{6}\right)^{\frac{3}{2}} \approx 1.139547}$ \\
\hline
\end{tabular}

Table 1. Statistical properties of Weibull and Gumbel distributions

The first three methods were implemented in the FITS program developed at Stanford University [15] and the fourth by a minor addition to this same program.

\section{Extreme Value Analysis}

The first part of this report will focus on extreme values, while the second part will focus on fatigue analysis. The estimate of the long-term behavior for these different loading types is similar. For example, both methods use thresholding to improve the accuracy of their short-term distribution fits. However, there are subtle differences which are highlighted in each section below.

\section{Selection of the threshold value}

Before short-term probability distributions of the extreme values could be calculated, the local maxima data (or local extremes) had to be extracted from the bending load time series. A peakover-threshold method was used to extract these data. This method selects the largest value between positive slope up-crossings of the threshold and also reduces the potential correlation between selected local maxima. Non-correlation is critical because the method used to calculate the long-term distributions assumes that the sampled local maxima are independent, as described below. This method also eliminates the majority of smaller amplitude extremes that are less significant when extrapolating to large amplitude long-term loads. 
Ideally, researchers would choose a threshold such that only the most statistically relevant extreme values remained in the distribution. Researchers could use personal judgment and visual inspection to identify the ideal threshold value. However, in this study, because we needed to identify 525 separate threshold values, a more automated approach was needed. The chosen method for determining the threshold was based on the mean and standard deviation of the original time series, as described below. More sophisticated methods for determining the threshold may produce better results, and this is an area for future study.

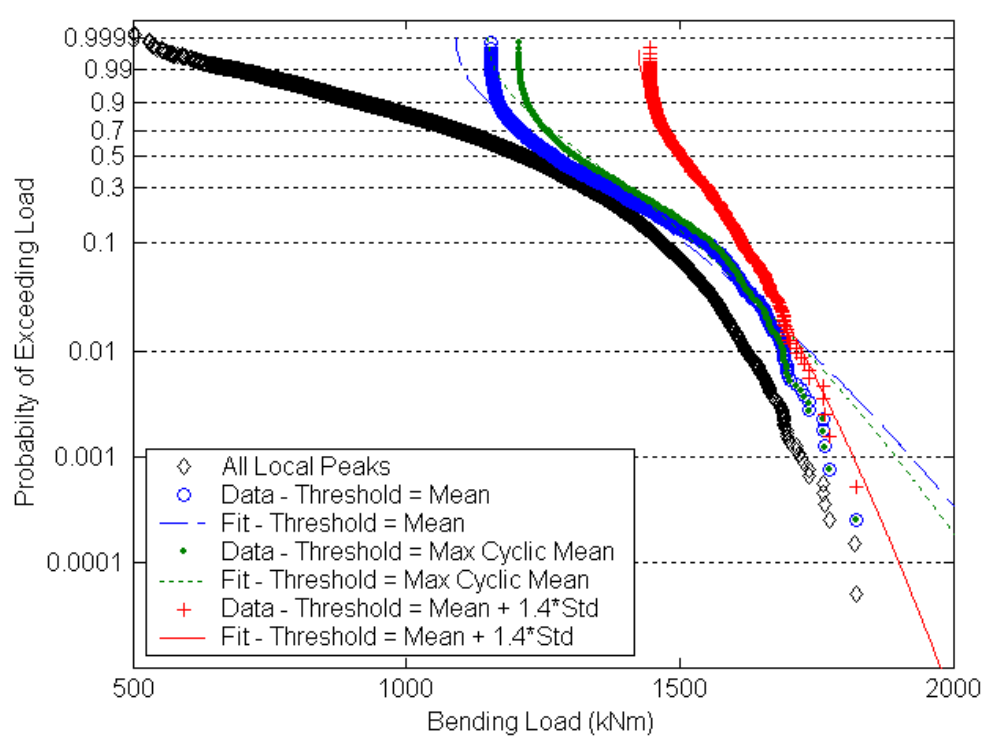

Figure 3. Effect of threshold on accuracy of fit for extreme values (Out-of-plane bending load of pitch-regulated turbine)

The choice of threshold has a significant effect on the statistics and shape of the distribution, as seen in Figure 3. This figure shows four different distributions of extremes and three resultant fits using three different threshold values and a three-parameter Weibull model. The data have all been extracted from the same original time series of out-of-plane bending load from a compilation of nine 10-minute simulations for the pitch-regulated machine. The wind conditions for this data set were a mean wind speed of $10 \mathrm{~m} / \mathrm{s}$ and a turbulence intensity of $18 \%$. In Figure 3, the three different thresholds examined were: the mean of the original aggregate time series data, the mean plus 1.4 times the standard deviation, and the maximum cyclic mean derived from azimuthal averaging of the time series.

The mean value is the lowest of these quantities and preserves the greatest number of maxima from the time series. Because much of the maxima data lies close to the threshold, however, the distribution is heavily weighted to lower values of the bending load and the fitted distribution does a poor job of following the trend of the tail of the data, or the highest values of bending load. It is common practice when extrapolating extreme values to concentrate on fitting the general distribution shape of the tail of the distribution since the largest values are of primary interest. Therefore, while using the mean of the time series data for the threshold preserves a large number of points and reduces the statistical uncertainty (as discussed in the next section), a higher threshold value should improve the accuracy of the fitted distribution in following the trends in the tail of the data.

The next higher threshold value is that of the maximum cyclic mean. This value was obtained by calculating the azimuth average of the aggregate time series in 36 different azimuth angle bins and finding the largest value. The maximum cyclic mean is slightly higher than the overall mean value for the out-of-plane bending load and therefore eliminates some of the lower value extremes. However, there is only a slight improvement of the fitted distribution in matching the tail of the data, as seen in Figure 3. 
The next highest threshold examined was the mean plus 1.4 times the standard deviation of the original time series. From Figure 3, we can see that this value is much higher than the previous two thresholds and many of the lower value extremes are removed from consideration. The distribution fit to the data much more closely follows the trend in the tail. We might expect that an even higher threshold would produce a fitted distribution that could almost perfectly match the largest data values. The largest values, however, exhibit considerable scatter compared to the tail of the distribution from which they are drawn. Thus, lower statistical uncertainty is obtained by retaining more data so long as it represents independent samples from the same parent distribution whose shape accurately follows the selected distribution shape. Also, for some time series a threshold of the mean plus 1.4 times the standard deviation was the largest value found that did not eliminate a majority (or all) of the local maxima. This is particularly important at low turbulence levels where the time series essentially becomes a deterministic sine wave whose maximum point is always the mean plus $1.414(\sqrt{2})$ times the standard deviation. These lowturbulence cases were considered of less importance in determining the overall exceedance probability. A threshold of the mean plus 1.4 times the standard deviation was found to be a good balance between low statistical uncertainty and concentrating on data that represent the trend for the distribution tail. This value of threshold was used to extract local maxima for all of the data sets.

Note in Figure 3 that as the threshold value increases so does the probability of exceedance of the data values, which is most noticeable on the tails of the distributions. This effect is due to the fact that the probability of exceedance for the largest data value in a set is inversely proportional to the number of data points in the data set, i.e. fewer data points results in higher minimum exceedance probability for a given data set time duration. To further demonstrate that this is the cause of this behavior, the distributions can be normalized relative to the number of data points. Figure 4 shows the normalized distributions of all the local maxima and the distribution using the highest threshold value, where the normalization factor is the total number of local maxima acquired without a threshold. The two curves collapse fairly well upon normalization, although not perfectly. For example, the second largest local maximum (probability just above 0.0001 ) in Figure 4 is not selected by the peakover-threshold method but remains in the distribution of all the local maxima. The elimination of this peak using a threshold method is appropriate since it occurred within $0.35 \mathrm{sec}$. of the largest peak in the original time series and thus would have been significantly correlated.

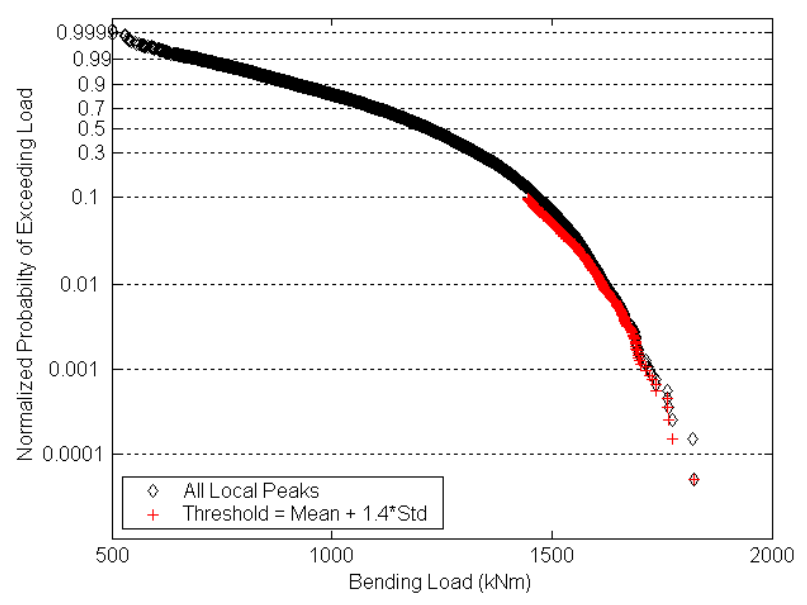

Figure 4. Normalized probability distributions of extreme values (Out-of-plane bending load of pitch-regulated turbine) 


\section{Uncertainty of the statistical moments}

One disadvantage of using a moment-based probability fitting technique, compared, for example, to a least-squares regression or maximum likelihood method, is that the statistical moments themselves contain a certain amount of statistical uncertainty that can significantly affect the shape of the distribution. In addition, the higher the order of the statistical moments used, the more uncertain these moments will be for a given data set with a fixed number of points. In order to examine the relative uncertainty of the statistical moments, an additional, more extensive, study at a single wind speed and turbulence level was performed. In this study, we evaluated the variation of the first three moments, the shifted mean (mean value - threshold), standard deviation and skewness, and two other quantities: threshold value and number of maxima per $10 \mathrm{~min}$. simulation. The data used in the study consisted of 300 10-minute time series simulations of the out-of-plane bending load at the root of the blade on the pitch-regulated turbine operating in a 10 $\mathrm{m} / \mathrm{s}$ mean wind with $18 \%$ turbulence intensity. Each of these time series was randomly grouped into an increasing number of aggregate simulations from which maxima were extracted and statistical moments were calculated. Fifty different random groupings of 1 to 100 simulations were examined and the threshold value, shifted mean, standard deviation, and skewness of the resulting maxima, as well as the number of maxima are shown in Figures 5-9 respectively.

The dashed horizontal lines in each of these figures represents $5 \%$ deviation (above and below) from the mean value of the 50 groups of the statistical moment with an aggregate file size of 100 simulations, i.e. the mean of the right-most points in each figure. This $5 \%$ deviation was found to have an effect on the long-term fully integrated probability distributions of extreme loads, where a $5 \%$ change in shifted mean value for all wind conditions leads to a relatively small $0.3 \%$ change in the predicted 50-year extreme load, while a 5\% change in the standard deviation results in a $2.8 \%$ change in the 50 -year load, and a $5 \%$ change in the skewness results in a $3.4 \%$ change in the 50-year load. Equivalent changes in threshold value results in a $2 \%$ change in the 50 -year load. And finally, a 5\% deviation in the number of maxima per 10 min. simulation would produce a minimal $0.2 \%$ change in the 50 -year load. Obviously, from these figures, the skewness is going to have the greatest effect on the predicted long-term load as it is not fully converged within the $+/-5 \%$ band for even 100 simulations. The standard deviation will also af-

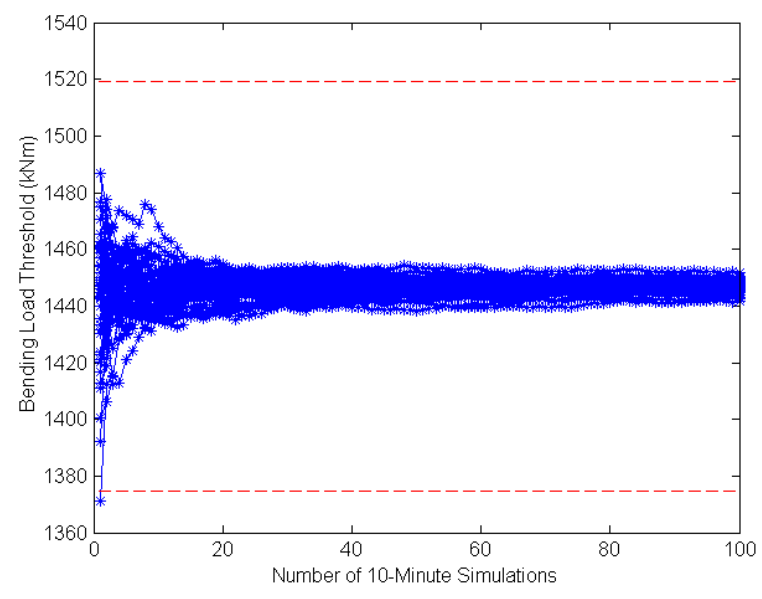

Figure 5. Calculated bending load threshold vs. number of aggregate 10 -minute simulations (Out-of-plane bending load of pitch-regulated turbine)

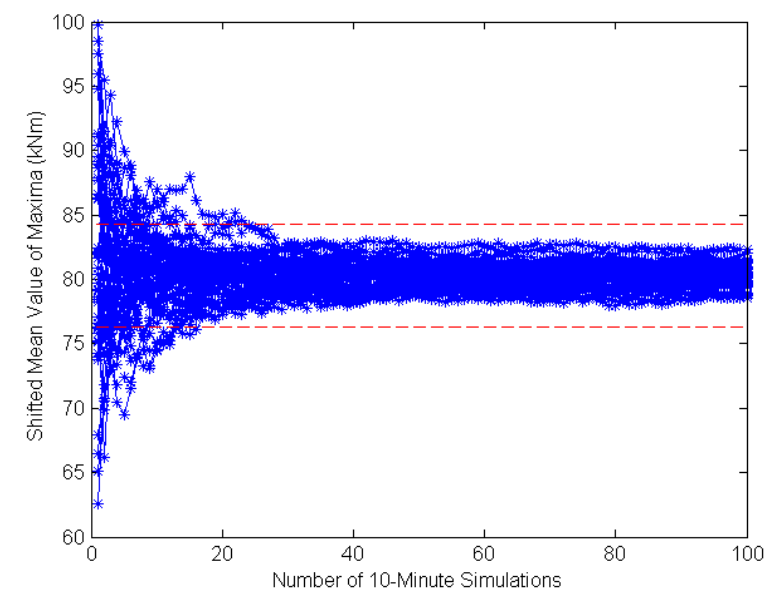

Figure 6. Calculated shifted mean value of maxima vs. number of aggregate 10 -minute simulations (Out-of-plane bending load of pitch-regulated turbine) 


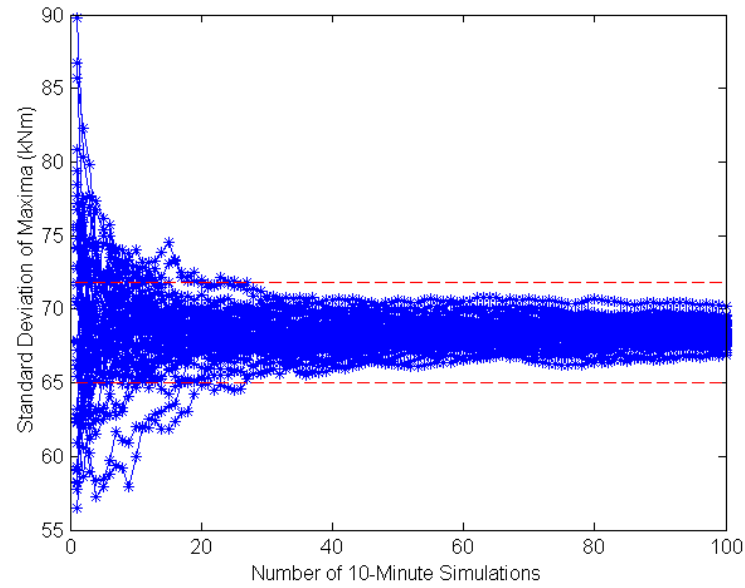

Figure 7. Calculated standard deviation of maxima vs. number of aggregate 10-minute simulations (Out-of-plane bending load of pitch-regulated turbine)

fect the long-term load if the number of simulations is less than 30 . Variation in the threshold value will not greatly affect the long-term load because it is fairly stable even for a very low number of simulations in the aggregate data set. Changes in the shifted mean and number of maxima per $10 \mathrm{~min}$. also will have little effect on the long-term load because of their low sensitivity.

From these figures, we can see that using nine simulations per wind condition is insufficient to provide stable moments for long-term probability distributions. Ideally, 30 or more simulations per wind condition would be available. However, with 525 wind conditions being modeled, the total number of simulations would quickly become prohibitive in terms of computation time.

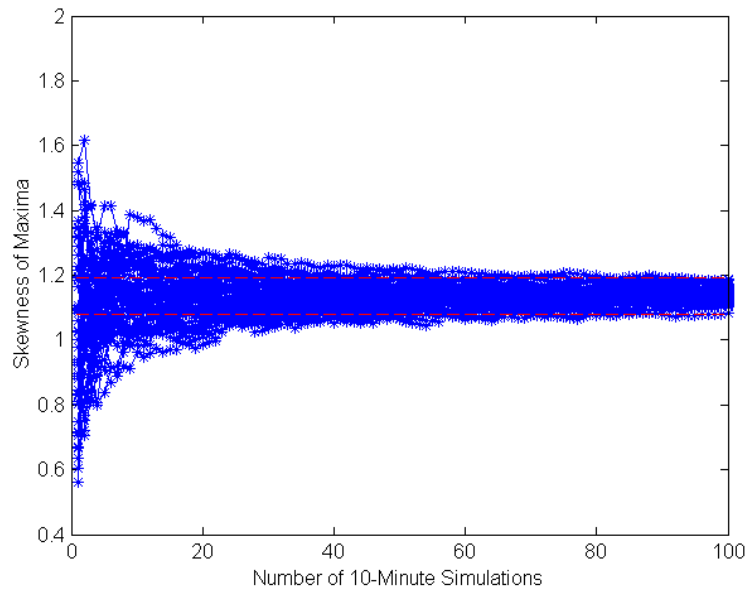

Figure 8. Calculated skewness of maxima vs. number of aggregate 10-minute simulations (Out-of-plane bending load of pitch-regulated turbine)

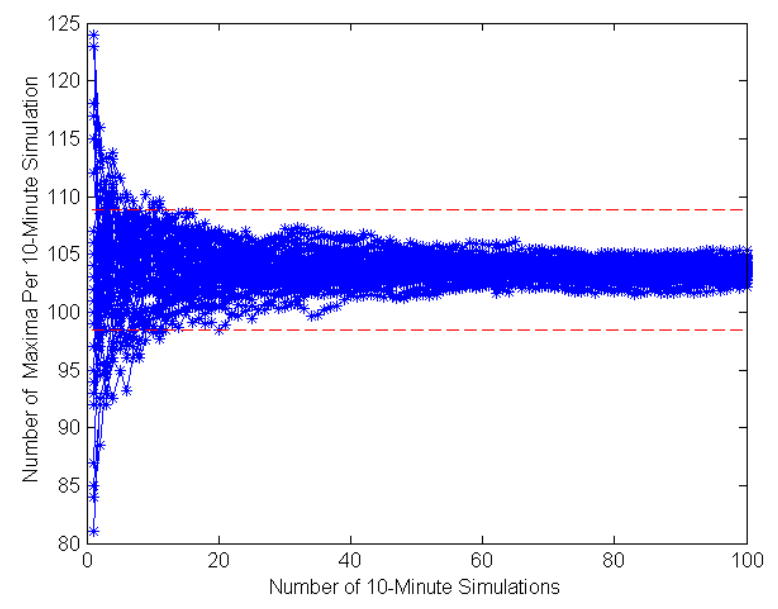

Figure 9. Calculated number of load maxima vs. number of aggregate 10 -minute simulations (Out-of-plane bending load of pitch-regulated turbine)

To increase the effective number of simulations per data point and reduce the statistical uncertainty of the moments, we investigated averaging statistical moments between various wind conditions. Figure 10 shows a plot of the skewness for the out-of-plane bending load maxima from the pitch-regulated turbine at all simulated wind conditions. There is some variation, particularly around the rated wind speed of $11 \mathrm{~m} / \mathrm{s}$, but a simple regression through all of these points reveals that the trend in the skewness is fairly constant with both wind speed and turbulence intensity. Figure 11 shows the skewness values for a stall-regulated turbine. These skewness values have even less variation than those of the pitch-regulated turbine and the data trend remains well approximated by a constant value. Therefore, for all long-term probability distributions the skewness of each short-term distribution was assumed to be a constant value equivalent to the mean skewness of all simulated wind conditions (the dashed lines in Figures 10 \& 11). For the other two statistical moments, mean and standard deviation, as well as threshold value and number of 


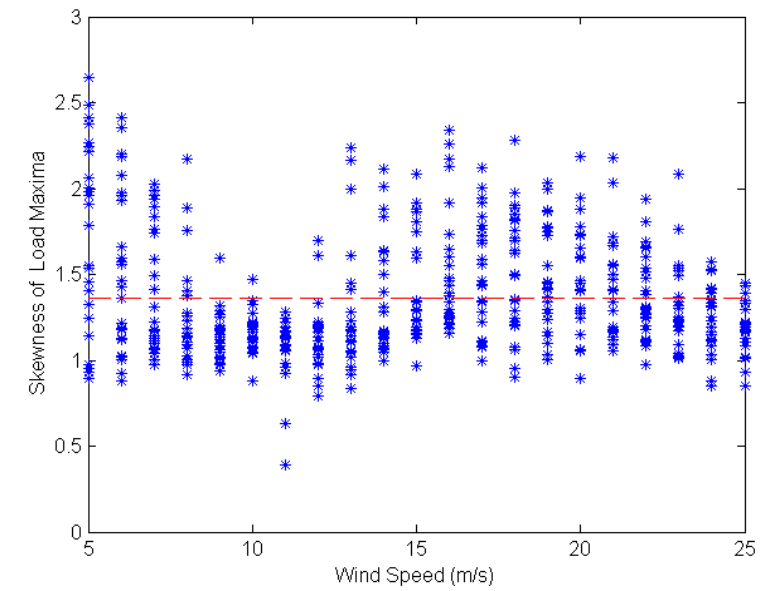

Figure 10. Skewness of load maxima for pitchregulated turbine (Out-of-plane bending load)

data points used to calculate the short-term distributions, a smoothing process was implemented. This smoothing process was done by combining an average of the statistical moment at each wind condition with each neighboring wind condition in both the mean wind speed and turbulence direction, i.e. the average of five wind conditions. This averaging lowered the statistical uncertainty of the moments, but had a fairly minor effect on the predicted long-term distribution, as seen in Figure 12. The reason the effect of smoothing is not more substantial is likely because the integrated long-term distribution tends to average out the random variations in the unsmoothed data when all wind conditions are considered.

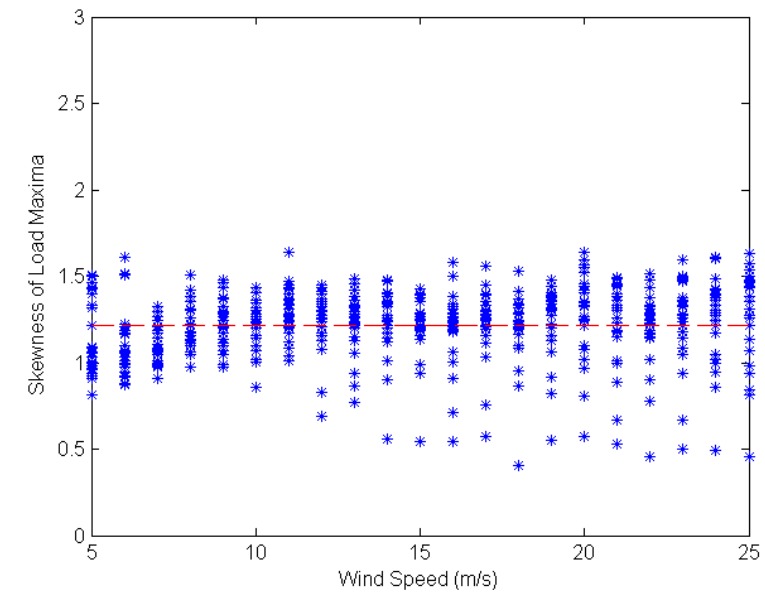

Figure 11. Skewness of load maxima for stallregulated turbine (Out-of-plane bending load)

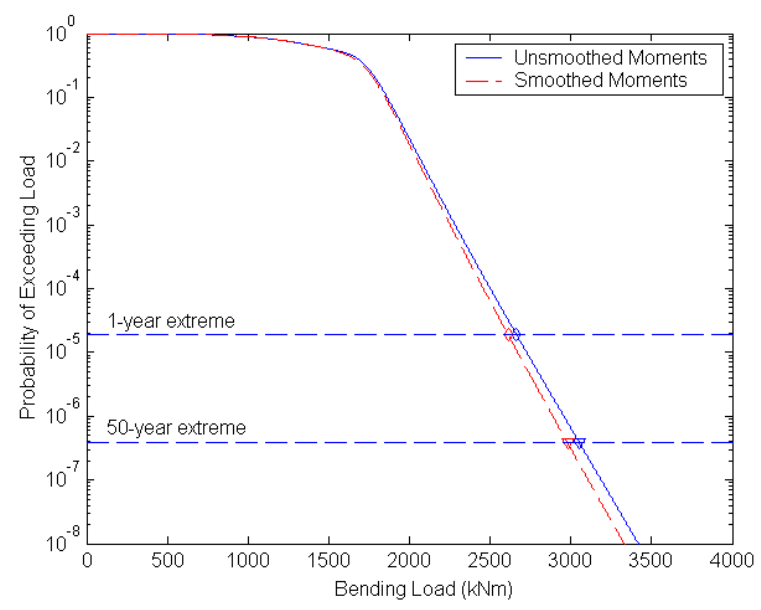

Figure 12. Effect of moment smoothing on long-term probability distribution for pitchregulated turbine (Out-of-plane bending load)

\section{Short-term probability fits}

Before a long-term distribution of extreme loads could be estimated, the short-term probability distribution was calculated for each $(\mathrm{V}, \sigma)$ combination. The choice of distribution used to model the short-term data distribution had a significant effect on the accuracy of the probability distribution in following the trends in the data, particularly for the largest data values (or tail of the data). The largest data values are considered most important for loads extrapolation because they are most likely to be physically and statistically similar to the long-term extrapolated load values.

Because the Weibull and quadratic Weibull methods are constrained to pass through zero moment, better fits result when the distribution starts near zero. However, the local extremes were extracted using a peak-over-threshold method, and hence no data existed below the threshold value. Therefore, before calculating moments and fitting the distribution, all data were shifted by subtracting the threshold value, which was the mean load from the combination of the nine 10min. simulations at each wind condition. Because of their formulation, the shift does not affect the Gumbel or three-parameter Weibull models. 


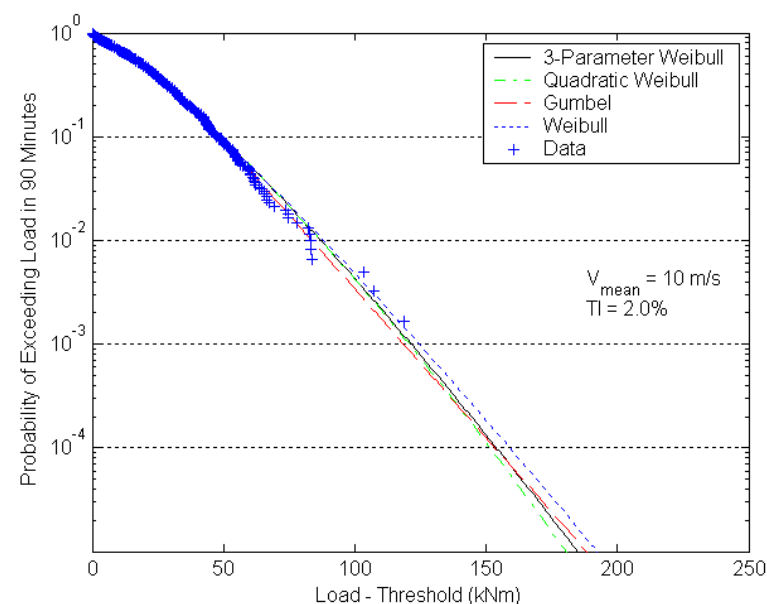

Figure 13. Probability of exceedance, $V_{\text {mean }}=10$ $\mathrm{m} / \mathrm{s}, \sigma=0.2 \mathrm{~m} / \mathrm{s}$, pitch-regulated turbine (Outof-plane bending load)

Figures 13-15 show the exceedance probability from these distribution fits along with the shifted data for out-of-plane bending load of the pitch-regulated machine. Each of these figures shows data at the same mean wind speed, 10 $\mathrm{m} / \mathrm{s}$, but at three different turbulence levels. Figure 13 shows the distributions for $2 \%$ turbulence intensity. The largest data values, out on the tail of the distribution, lie very close to the lines for all of the fitted distributions and therefore no distribution model is obviously more accurate for this wind condition. Figure 14 shows the distribution for a more common turbulence intensity of $18 \%$. In this plot, there is a slight kink in the shifted data near $200 \mathrm{kNm}$ that is not properly modeled by any of the fitted distributions. The quadratic Weibull and threeparameter Weibull are the closest distributions to the trends in the data, but still overpredict the load for a given level of probability. This kink indicates that there is a change in the physical process for higher load values, which may require more complex models to properly follow the probability distribution. The data from simulations at a larger turbulence intensity of 50\%, seen in Figure 15, also appear to diverge from each of the fit models, with an even more pronounced kink than the $18 \%$ turbulence intensity. Again, the closest fit to the data in this figure is the quadratic Weibull model. If a long-term load were extrapolated using these short-term fits, one would expect that the extrapolated value would overpredict the true load.

Interestingly, as shown in Figure 16, the divergence between the data and the fitted distributions is not a problem for the stall-regulated turbine. For the same wind conditions present in Figure 15, the fitted distributions of the stall-regulated turbine follow the trends in the tail of the data, which does not contain the kink seen in the pitch-regulated machine data at higher turbulence intensities. Further examination of several data sets from the pitch-regulated turbine indicates 
that this divergence is particularly prevalent around rated wind speed $(11 \mathrm{~m} / \mathrm{s})$. It is likely that near rated wind speed, the control system of the pitch-regulated turbine changes operating regimes many times during a $10-\mathrm{min}$. simulation. This is more likely to occur at higher turbulence intensities, which may explain the kink in the data that cannot be modeled by the method of moments. This should be an area of future research and may require an alternative method of fitting the probability distributions of pitch-regulated turbine loads.

Comparing the behavior seen in these plots, it is not obvious which probability model should be used to fit the maxima data. It is clear, however,

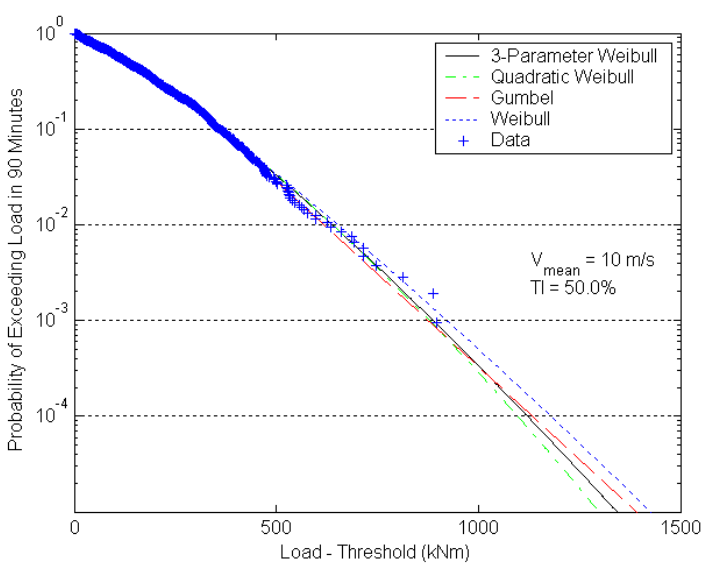

Figure 16. Probability of exceedance, $V_{\text {mean }}=10$ $\mathrm{m} / \mathrm{s}, \sigma=5.0 \mathrm{~m} / \mathrm{s}$, stall-regulated turbine (Out-ofplane bending load) that the turbulence intensity has a significant effect on the statistics of the distribution. This is somewhat intuitive, as the process becomes less deterministic when the amplitude of the stochastic component (i.e. turbulence) increases and vice versa. Another important trend to note in these figures is the relative tail behavior of the three distribution fits. At low probabilities of exceedance, the regular Weibull distributions will consistently predict the largest values, followed by the value using a Gumbel, then three-parameter Weibull, and finally the smallest value with a quadratic Weibull.

\section{Long-term extreme loads extrapolation}

Once probability distributions to the short-term data have been computed for each wind condition, a long-term exceedance distribution can be computed by integrating all of the short-term distributions with the joint probability density function.

$$
P\left[L_{10 \text { min. }}>L\right]=\int_{V_{\text {in }}}^{V_{\text {our }} \infty} \int_{0}^{\infty} P\left[L_{10 \text { min. }}>L \mid V, \sigma\right] f_{V, \sigma}(V, \sigma) d \sigma d V
$$

where $f_{V, \sigma}(V, \sigma)$ is the joint probability density function of the wind conditions. Before doing so, it is also important to transform the short-term probabilities from those of local maxima to the probability distribution for the single largest event in 10 minutes. Assuming that the local maxima are statistically independent, this probability can be estimated as

$$
P\left[L_{10 \text { min. }}<L \mid V, \sigma\right]=\left(P\left[L_{\text {local } \max }<L \mid V, \sigma\right]\right)^{N}
$$

where $N$ is the total number of local maxima occurring in a 10 min. period. Note that the number of maxima changes with the turbulence level and wind speed due to the fact that the mean crossing frequency increases with turbulence intensity and wind speed. Because of this, the number of maxima must be calculated or estimated for each $(\mathrm{V}, \sigma)$ combination.

Once the integration of short-term distributions is complete, extrapolated loads from the resulting exceedance distribution can be estimated. Figure 17 shows the long-term distribution for the outof-plane loads of the pitch-regulated turbine. Again, four different distribution fits are shown; each of them was calculated using a log-normal turbulence distribution. The two horizontal lines 


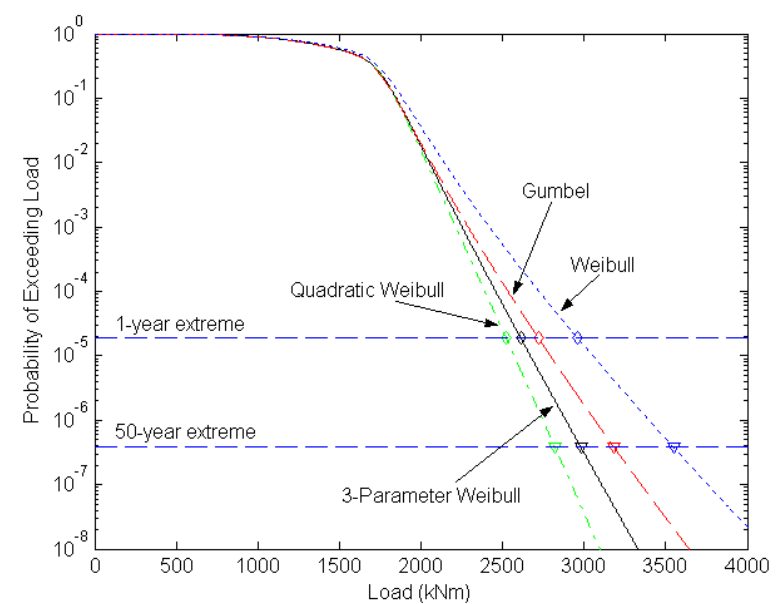

Figure 17. Long-term exceedance probability distributions for out-of-plane load of the pitchregulated turbine

in Figure 17 represent the 1- and 50-year exceedance probability level, with values of $1.9 \times 10^{-5}$ and $3.8 \times 10^{-7}$ respectively. The point at which the distributions cross these lines are the estimated 1- and 50-year extreme loads. Similar to the trend seen in the short-term distributions, the regular Weibull model predicts the highest extreme loads followed by the Gumbel, threeparameter Weibull, and then quadratic Weibull. Figure 18 shows the out-of-plane distributions for the stall-regulated turbine. The trend in predicted load vs. distribution choice is identical to the pitch-regulated machine, but the variation in predicted loads is much smaller. This smaller variation is most likely due to more accurate fitting of the data in the short-term distributions of the stall-regulated machine.

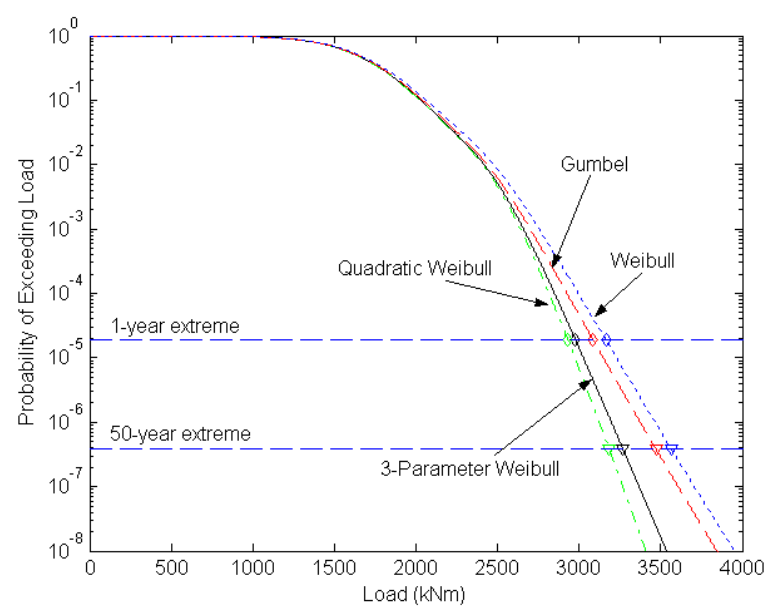

Figure 18. Long-term exceedance probability distributions for out-of-plane load of the stallregulated turbine

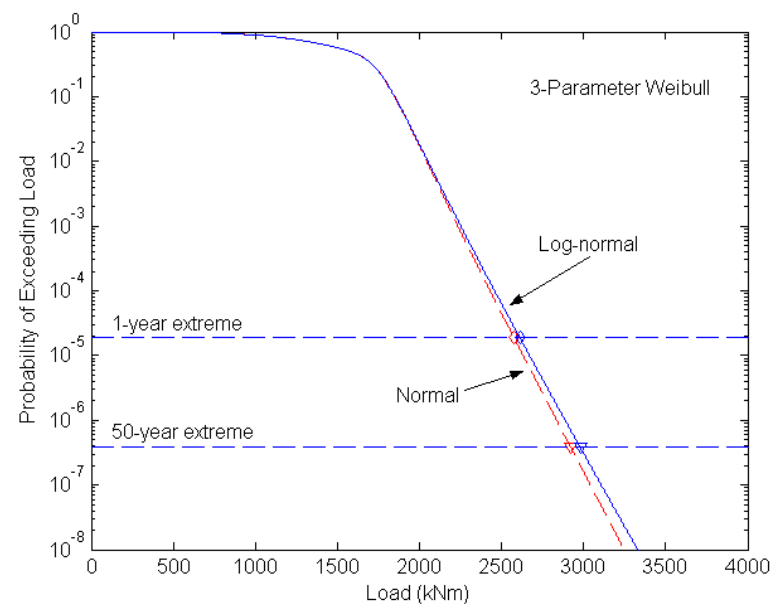

Figure 19. Comparison of effect of turbulence distribution on long-term exceedance probability for out-of-plane loads of the pitch-regulated turbine

Upon detailed examination of the statistical

moments, we found that some of the distributions of load maxima had negative skewness values. For Weibull and Gumbel distribution-based models this presents a problem because a negative skewness is not represented by the model. Only the quadratic Weibull model is capable of yielding a negative fitted skewness and only for severe distortion values. Therefore, any data set with negative skewness was neglected in the long-term probability calculation. Because these cases occurred for low probability, high-speed, low-turbulence wind conditions, ignoring them had a negligible influence on the predicted long-term load.

Figure 19 shows a comparison between using a normal and log-normal turbulence distribution for the pitch-regulated machine. The choice of distribution has little effect on the predicted 1year extreme load and has a minor effect on the 50-year load. At lower probability levels, the difference increases, but is never greater than 5\%. The three-parameter Weibull distribution, out of the four models examined, was found to have the largest differences between turbulence dis- 
tributions. The other probabilistic fits had similar behavior at low probability levels, but the differences were smaller.

\section{Model simplification}

While using the integration method along with simulations at all probable wind conditions is thorough, the number of simulations required may not be practical from a design perspective due to time constraints or limited amounts of data available from experiments. Because of this, several alternatives to the integration method are proposed to reduce the amount of data required for extrapolation. These methods are input extrapolation, parametric modeling, and deterministic turbulence modeling.

\section{Input extrapolation}

One way to decrease the amount of simulated data needed is to extrapolate the wind inputs before running them through the aeroelastic simulations. A simple method for extrapolating inputs involves finding the 1- and 50-year extreme turbulence level at each mean wind speed. This is done by renormalizing the conditional density function of the turbulence level to produce an exceedance plot of turbulence level at each wind speed, assuming 100\% operation at each wind speed. Figure 20 shows an example of this procedure for $\mathrm{V}_{\text {mean }}=10 \mathrm{~m} / \mathrm{s}$. The 1 - and 50 -year extreme turbulence levels were estimated from this plot and simulations were performed using $\left(\mathrm{V}_{\text {mean }}, \sigma_{1}\right)$ and $\left(\mathrm{V}_{\text {mean }}, \sigma_{50}\right)$ as inputs. The largest value of the bending load from the output of this simulation is then the extreme load at this mean wind speed. This process is repeated for all operating wind speeds and the largest value for all wind speeds is the extreme load. Since the number of turbulence levels at each mean wind speed is reduced to 2, the simulation time savings are significant.

To check the validity of this method, nine 10-min. simulations were run for each $\left(\mathrm{V}_{\text {mean }}, \sigma_{1}\right)$ and $\left(\mathrm{V}_{\text {mean }}, \sigma_{50}\right)$ combination and the extreme values were extracted from each simulation. An average value of the extremes was then calculated at each wind condition to reduce the statistical uncertainty of the predicted extreme value. The predicted extreme values of the out-of-plane load for the stall-regulated turbine from each simulation and the average at each mean wind speed us-

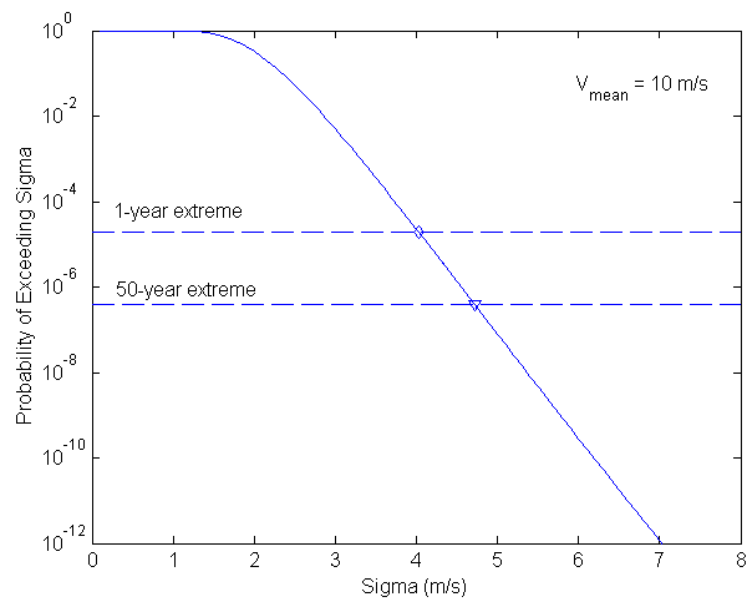

Figure 20. Exceedance probability for turbulence levels at $V_{\text {mean }}=10 \mathrm{~m} / \mathrm{s}$

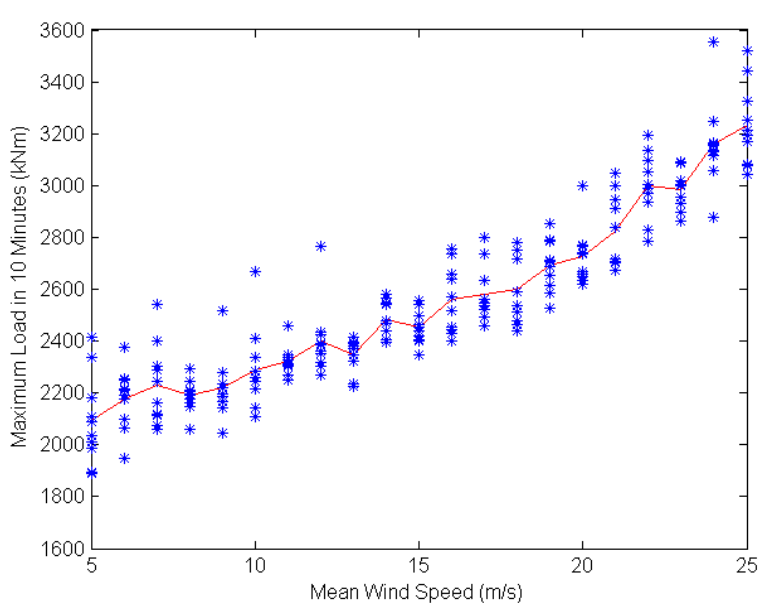

Figure 21. Extreme value in $10 \mathrm{~min}$. simulation using extrapolated 50-year turbulence level (Out-of-plane bending load of stall-regulated turbine) 
ing the 50-year turbulence level are shown in Figure 21. Using this method, the predicted 50year load is approximately $3200 \mathrm{kNm}$. Comparing this result to the full integration method of Figure 18, we see that the predicted load is very close to that predicted by the three-parameter Weibull and quadratic Weibull models.

One disadvantage of this technique is that it is sensitive to the choice of cut-out wind speed. For a stall regulated machine, an increase in cut-out wind speed also leads to a comparable increase in predicted extreme loads. The sensitivity for a pitch regulated machine is probably less, since the largest loads tend to occur near the rated wind speed for these machines.

This input extrapolation scheme is a simplified version of a technique known as the Inverse First Order Reliability Method (or inverse FORM). Several recent studies of inverse FORM [17, 18] have shown its usefulness for wind turbine applications. The authors will defer to the listed references for a more detailed explanation of this useful design tool.

\section{Parametric modeling}

Another method for reducing the amount of data required that can also reduce the uncertainty of the statistical moments is to parametrically model the moments. In past studies $[2,8,10]$, power law parametric models have been used to represent the various statistical moments with fairly good results. In one of the studies [10], the parametric model was also used to calculate the number of load maxima and the load threshold for a stall-regulated turbine. In this study, using a power law to model the load threshold of a pitch-regulated turbine proved to be much more difficult. As shown in Figure 22, the load threshold (mean value plus 1.4 times standard deviation of the original time series) is a highly nonlinear function of mean wind speed that peaks at rated wind speed and falls off dramatically on either side. The variation of the threshold value with turbulence also depends upon mean wind speed. The threshold value is fairly constant with turbulence near rated, but varies greatly with turbulence away from rated wind speed. Note that the threshold value is dependent upon turbulence level because the standard deviation of the bending load will be correlated to the standard deviation of the wind speed. Because of this complex behavior, an alternative parametric model is proposed that is linear with turbulence level and estimated independently for each integer value of mean wind speed as given in the following formula:

$$
\mu_{i}(V, \sigma)=a_{i}(V)+b_{i}(V)\left(\frac{\sigma}{\sigma_{r e f}}\right)
$$

Where $\mu_{i}(i=1-3)$ are the first three moments of the distribution, the bending load threshold, or the number of load maxima per $10 \mathrm{~min}$. simulation. The variables $a_{i}$ and $b_{i}$ are the corresponding undetermined coefficients at each mean wind speed, $V$. For this study we chose $\sigma_{r e f}=2.5 \mathrm{~m} / \mathrm{s}$. Each of the coefficient sets was determined using a linear least-squares regression technique.

Figure 22 also shows the resulting parametric data fit, shown as the curves for each wind speed, to the raw values, represented by the "+" symbols, computed for the threshold data. The parametric model follows the trends in the data as a function of mean wind speed and turbulence level quite nicely. Figures 23-25 show the parametric fit and data for the shifted mean, the standard deviation, and number of load maxima respectively. Again, the parametric fit of the data follows the trends nicely, although the fit for the number of load maxima is not quite as good for some turbulence levels at the higher wind speeds. Fortunately, the long-term probability distributions are fairly insensitive to this parameter. Note that a similar plot is not shown for the skewness as it 


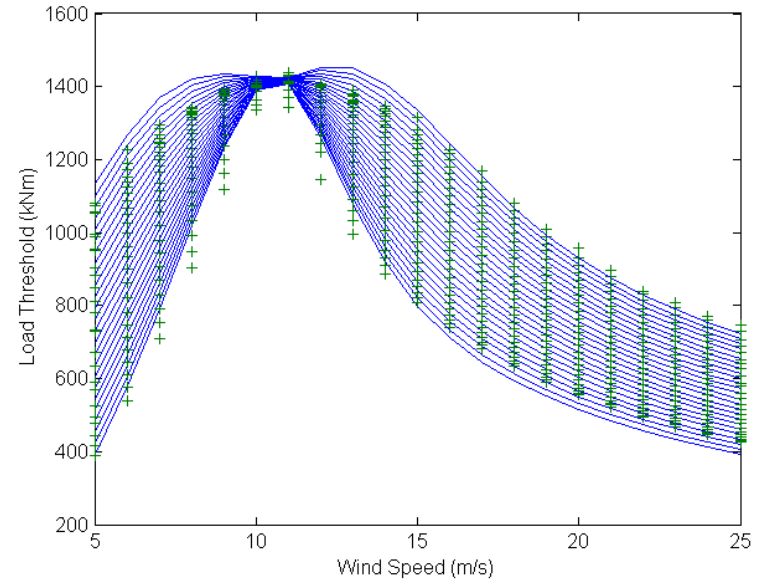

Figure 22. Load threshold value compared to parametric data fit (Out-of-plane bending load of pitch-regulated turbine)

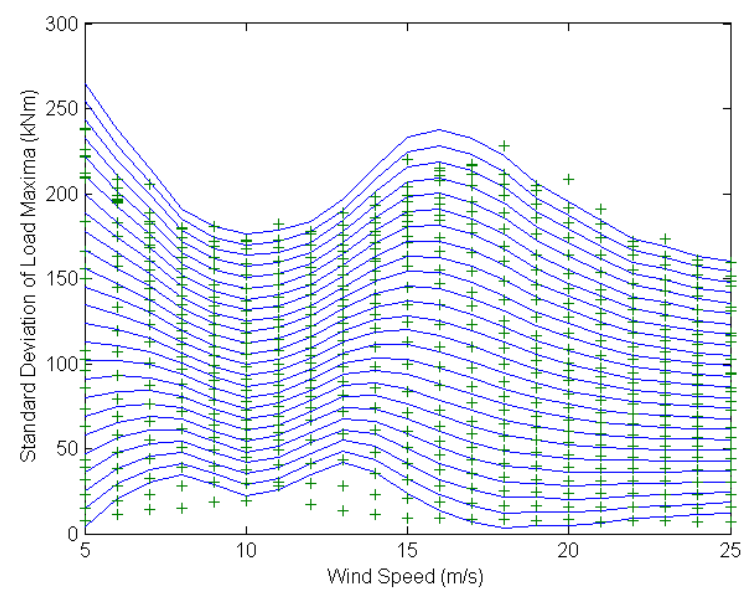

Figure 24. Standard deviation of load maxima compared to parametric data fit (Out-of-plane bending load of pitch-regulated turbine)

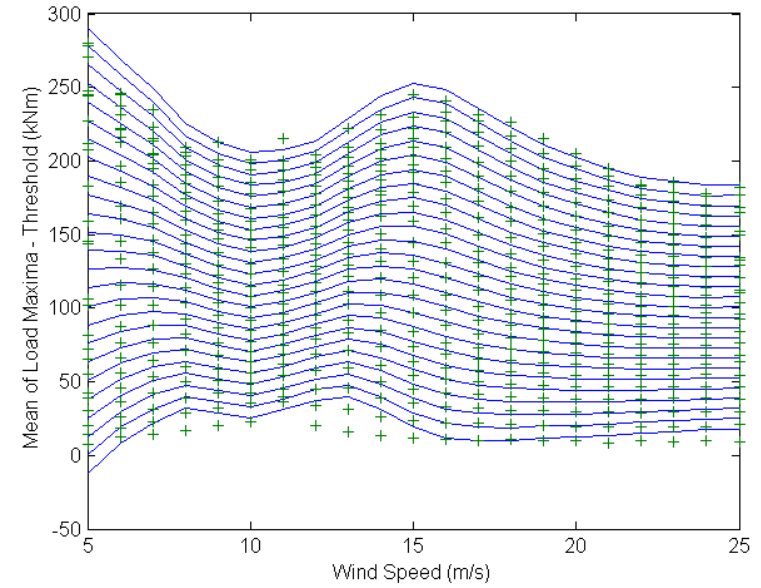

Figure 23. Shifted mean value of load maxima compared to parametric data fit (Out-of-plane bending load of pitch-regulated turbine)

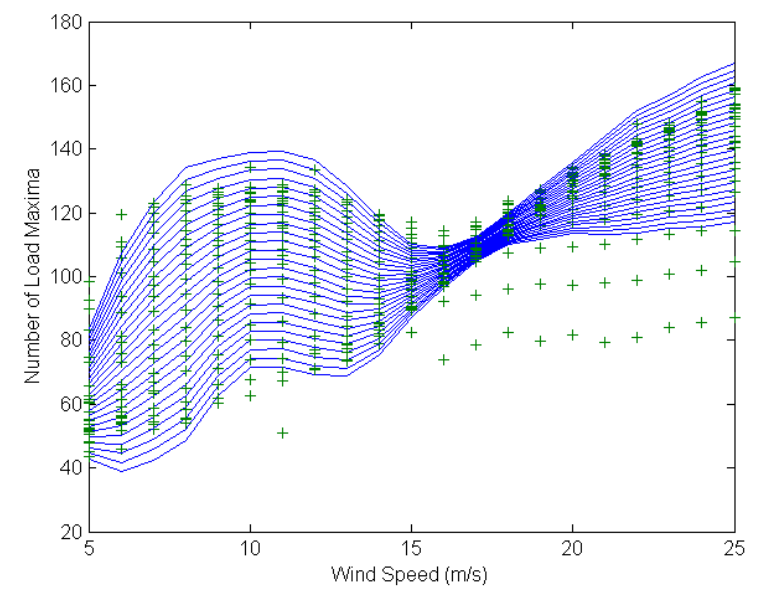

Figure 25. Number of load maxima compared to parametric data fit (Out-of-plane bending load of pitch-regulated turbine)

was fixed to a single value of the mean skewness for all wind conditions, as shown in Figures 10 and 11 .

Figure 26 shows a comparison of the fully integrated long-term probability distribution using both the empirical model of the data shown in Figures 22-25 and the equivalent parametric model for both in-plane and out-of-plane loads of the pitch-regulated turbine. Note that the outof-plane distribution using the empirical model in this figure is identical to the three-parameter Weibull distribution of Figure 17. For the in-plane bending load, the empirical and parametric results give identical probability distributions as well as predicted long-term loads. The out-ofplane load shows some differences between these two models, but the predicted long-term loads are within $5 \%$ of each other, with the empirical model being more conservative.

Figure 27 shows an equivalent comparison of the empirical and parametric models for both bending loads of the stall-regulated turbine. In this plot, the parametric and empirical models agree perfectly for both in-plane and out-of-plane loads and produce equivalent long-term loads. 


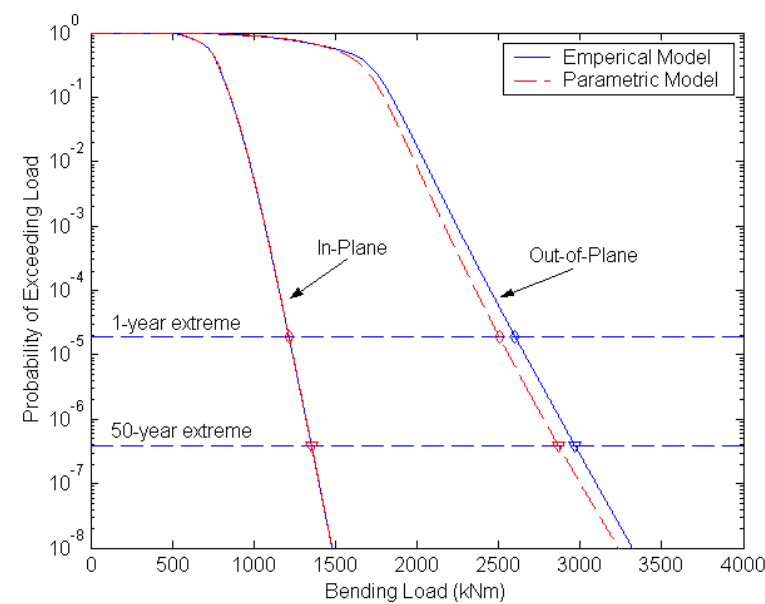

Figure 26. Long-term probability distributions from empirical vs. parametric models for a pitch-regulated turbine

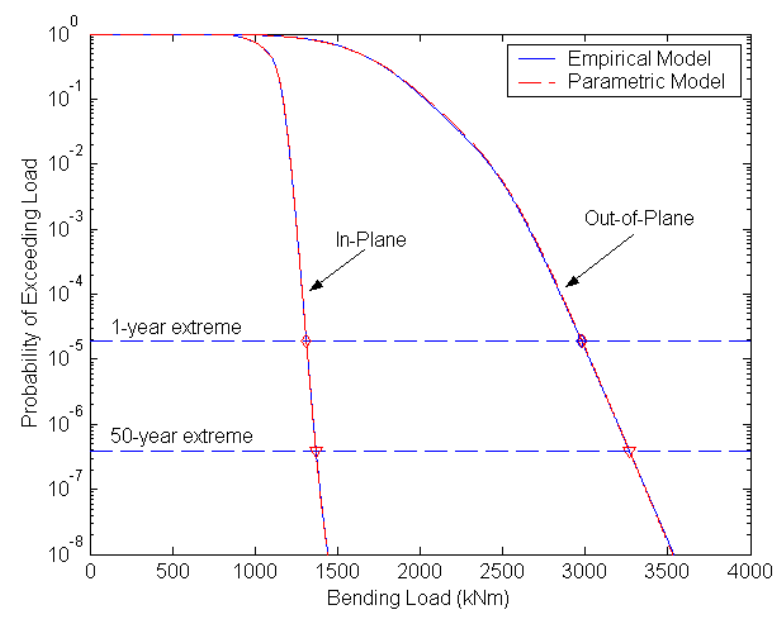

Figure 27. Long-term probability distributions from empirical vs. parametric models for a stall-regulated turbine

\section{Deterministic turbulence model}

Another technique of input extrapolation is to artificially increase the turbulence level for a given mean wind speed in the hope that it will produce loads similar to the full integration method. In this work, we propose that the turbulence level be assigned a deterministic value of the 90th percentile level calculated from the original log-normal distribution. This procedure results in a fairly simple equation for turbulence level given by

$$
\sigma(V)=I_{r e f}(0.8 V+b)
$$

where $I_{r e f}$ and $b$ are equivalent to parameters specified in the IEC $61400 \mathrm{Ed}$. 2 for the standard classes [1]. For class I-A, the values of these parameters are 0.15 and 6 respectively. Note that the $I_{\text {ref }}$ parameter has been chosen to represent a mean value rather than the mean plus one standard deviation given by $I_{15}$ in Ref. [1].

By using a deterministic turbulence level, one level of integration can be eliminated from the long-term probability calculation. Hence, Equation 32 becomes

$$
P\left[L_{10 \text { min. }}>L\right]=\int_{V_{\text {in }}}^{V_{\text {out }}} P\left[L_{10 \text { min. }}>L \mid V\right] f_{V}(V) d V
$$

Again nine simulations per wind condition were calculated and smoothing of moments was done between wind speeds, with skewness being fixed at the mean value for all wind speeds. Because only a single turbulence level was examined at each mean wind speed, the size of total data set required was 189 simulations instead of 4725 simulations for the full distribution method, resulting in a considerable time savings.

Figures 28 and 29 show the results of using the deterministic turbulence level to calculate the long-term probability distributions for both the pitch and stall regulated machine. Both figures show that the deterministic turbulence level predicts nearly identical long-term loads to the full integration method for the in-plane bending load. For the out-of-plane bending load the deterministic turbulence level predicts slightly higher extreme loads, where the differences for the pitch- 


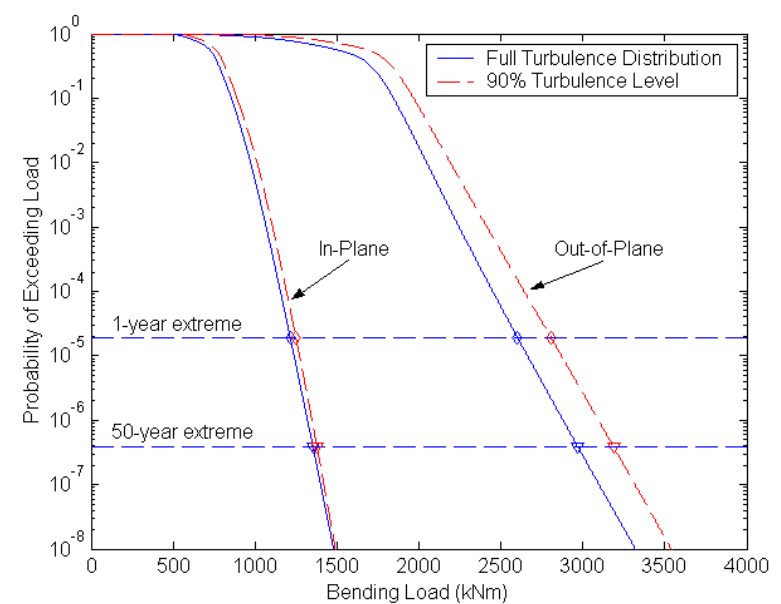

Figure 28. Comparison of predicted extreme loads for a pitch-regulated turbine using different turbulence distributions

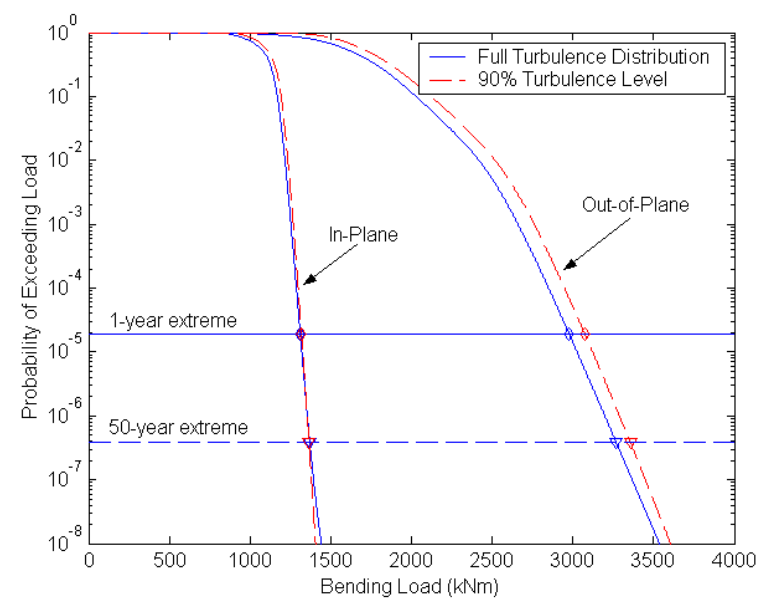

Figure 29. Comparison of predicted extreme loads for a stall-regulated turbine using different turbulence distributions

regulated turbine, shown in Figure 28, are nearly 10\%. If these loads are design drivers, this difference may motivate the designer to calculate the extreme loads using the more rigorous, fullintegration method and reduce the predicted extreme loads. However, one should realize that the level of conservatism depends on the sensitivity of the specific turbine and control system to turbulence in the inflow. The examples shown here reflect results applicable to the two turbines studied. Other turbines could exhibit higher or lower levels of conservatism.

\section{Fatigue}

The extrapolation of fatigue cycles is similar to that of extreme loading. Short-term distributions are fit to data at different wind conditions, after which long-term distributions are calculated using an integration method. An elevated turbulence intensity distribution can also be used to simplify the full-integration procedure. Fatigue extrapolation also contains some unique features unrelated to extreme extrapolation techniques, such as correcting fatigue amplitudes for cyclic midpoints and lifetime prediction. The details of these procedures and the usefulness of fatigue extrapolation are highlighted below.

\section{Cycle midpoint correction}

For wind turbine applications, the midpoint (or mean) of each rainflow-counted cycle is typically not equal to zero as is often assumed when calculating fatigue damage from a single S-N curve. Because a nonzero midpoint moves the maximum value in a cycle closer to the ultimate material strength, a midpoint offset can add significant damage for each cycle that must be estimated for a reliable life prediction. In order to compensate for a nonzero midpoint load, a Goodman correction was used to adjust the amplitude of the cycle [19]. The applicability of the Goodman correction differs with material properties, but for this study, provides a rough correction for the effect of a cyclic midpoint. This fit is assumed to collapse the S-N curves for different midpoint values. A typical Goodman correction adjusts any combination of alternating and midpoint loading to an equivalent, zero-midpoint alternating load. However, for large midpoint values, this approximation can lead to a significant distortion of the alternating load and could possibly change the tail of the distribution such that its statistical properties are physically incorrect. Instead, a Goodman 
correction can be used to adjust each cyclic amplitude to a fixed midpoint value closer to the original midpoint of each cycle using the following formula

$$
L_{a_{e q}}=L_{a}\left[\frac{L_{u l t}-L_{m_{e q}}}{L_{u l t}-L_{m}}\right]^{c}
$$

where $L_{a}$ and $L_{m}$ are the original load amplitude and midpoint, $L_{a}$ eq and $L_{m}$ eq are the equivalent amplitude and midpoint, $L_{u l t}$ is the ultimate strength, and $\mathrm{c}$ is the Goodman exponent, which was assigned the value of 1 . In this study, the equivalent midpoint value chosen was the overall mean load considering all simulations.

The ultimate strength for each turbine was assumed to be the 50-year extrapolated extreme load times a safety factor of 1.35 (as specified by the IEC [1]). These extrapolated extreme loads were equivalent to the extreme loads predicted by the three-parameter Weibull in Figures 17 and 18. The ultimate design loads were $4100 \mathrm{kNm}$ for the pitch-regulated turbine and $4550 \mathrm{kNm}$ for the stall-regulated turbine. The fatigue lifetimes resulting from this assumption provide a reference for determining whether fatigue or ultimate loads will govern the design. For estimated lifetimes significantly shorter than a desired lifetime, fatigue considerations will govern the design and there will be some margin in ultimate strength.

\section{Choice of threshold}

As with extreme values, when fitting a distribution of fatigue amplitudes it is more accurate for estimating the fatigue damage to fit the general trend of the tail of the data, i.e. the largest cyclic amplitude values. This is because the largest amplitude cycles tend to dominate the fatigue damage calculation [19] and any extrapolation of fatigue amplitudes to larger values should be based on the trend in the tail, whose data are assumed to be more statistically similar to the most damaging loads than the small amplitude data. Unfortunately, the process of rainflow cycle counting often produces many cycles with relatively small amplitudes as seen in Figure 30.

In this figure there are three sets of data: two are rainflow counted cycles from an aggregate of 300 simulations of the pitch-regulated turbine out-of-plane bending load at a mean wind speed of $10 \mathrm{~m} / \mathrm{s}$ and turbulence intensity of $18 \%$, while the other data set is an aggregate of only nine simulations (appropriately labeled) for the same turbine and wind conditions.

For each data set there is also a fit to the data calculated using the moment-based probability code. All short-term fitted probability distributions of fatigue cycles were calculated using a modified version of the FITS code [15]. All of the fits used a 3-parameter Weibull model, which required knowledge of the mean, standard deviation, and skewness of the data.

Concentrating first on the data set from 300 simulations, notice that the fit to the data is in-

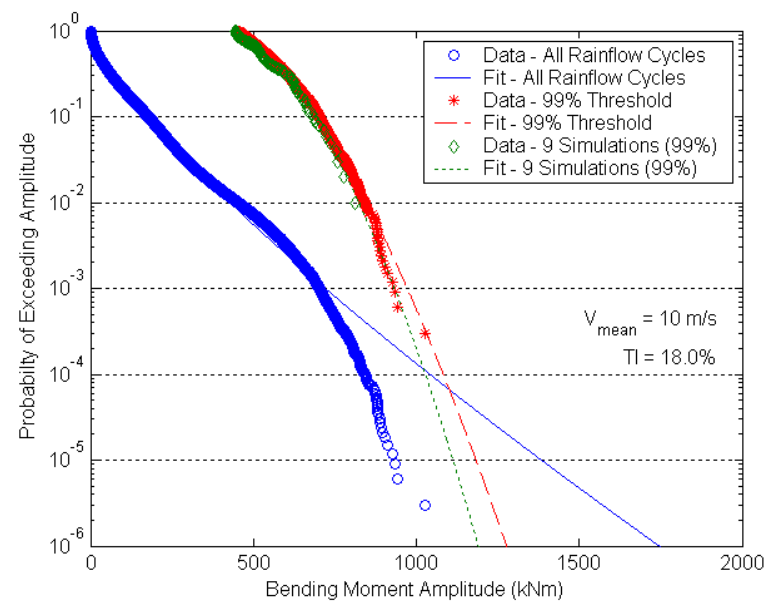

Figure 30. Threshold effect on exceedance probability and fitted distribution for out-ofplane bending load on the pitch regulated turbine. 
fluenced by a large number of small amplitude cycles and therefore deviates from the trend in the tail of the data. In order to improve the fit to the data, a threshold value for the fatigue amplitudes is used. All fatigue amplitude values above this threshold are retained and a fitted distribution is calculated only for these cycles. A reasonable threshold value for most wind conditions was found to be the 99th percentile of the cyclic amplitude distribution. Figure 30 also shows the distribution and fit to the data above the threshold. The agreement between the fit and the trend in the tail is significantly improved compared to using no threshold.

Because we examined 525 different wind conditions, it would have been unrealistic to run 300

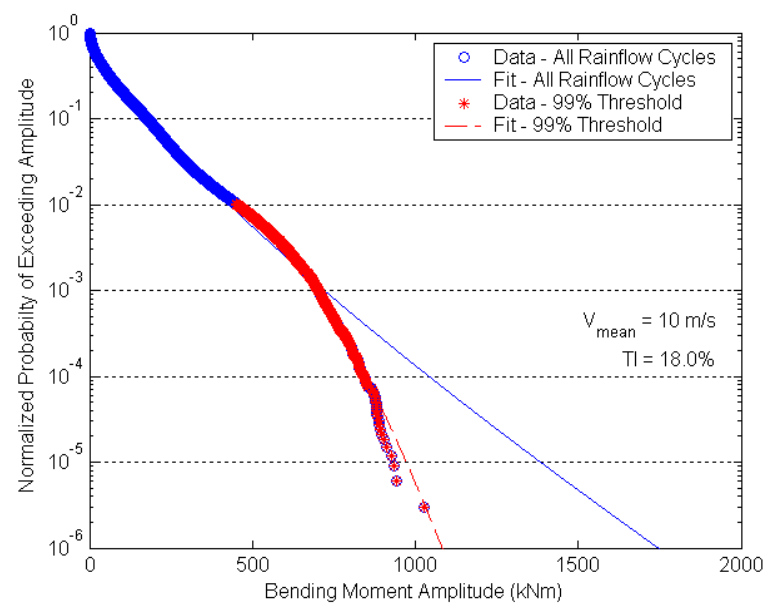

Figure 31. Normalized probabilities of exceedance for out-of-plane bending load on the pitch regulated turbine. simulations for each wind condition. Therefore, we limited the number of simulations per wind condition to nine. The third data set in Figure 30 shows the fatigue cycle amplitudes above threshold from an aggregate of nine simulations. Notice that the probabilities of exceedance are very similar to the larger data set, which gives some confidence that the limited data set at each wind condition will provide statistically similar results even though the number of data points is considerably less than the larger data set.

In Figure 30, the probability of exceedance for the data above the threshold is much different from the data without a threshold. As was demonstrated with the extreme values, this is because the probability of exceedance is inversely proportional to the number of data points in the data set for the fixed total time duration. After thresholding the data, there are many fewer points and hence the probability of exceedance for the same given duration is higher. If we normalize the probabilities by the number of points in the original data set, as shown in Figure 31, there is perfect agreement between the original data distribution and the data above the threshold. The normalized distributions also highlight the difference between the fits to the two different data sets.

Because the majority of fatigue cycles are removed when calculating the short-term distribution, using these fitted distributions to calculate damage can lead to non-conservative results. This is particularly true when the material fatigue exponents are low and the largest fatigue cycles no longer dominate the accumulated damage. In order to compensate for this effect, after thresholding the amplitude cycles the damage for all of the cycles below the threshold was calculated for each wind condition. This damage was then added to the damage calculated from the fitted distribution in order to calculate fatigue lifetimes, as described below.

\section{Long-term cycle distribution}

After correcting for varying midpoints and thresholding the data at each wind condition, longterm cyclic distributions were determined. Similar to the method used for extreme values, the cyclic distributions were calculated by integrating the short-term probabilities at each wind condition with the joint probability density function and the expected number of cycles as shown in the following formula 


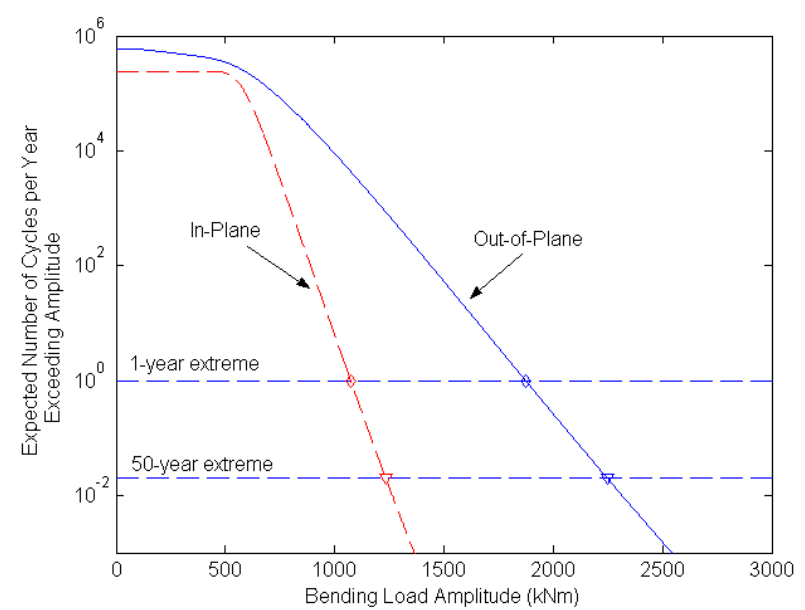

Figure 32. Expected number of cycles per year for pitch-regulated turbine.

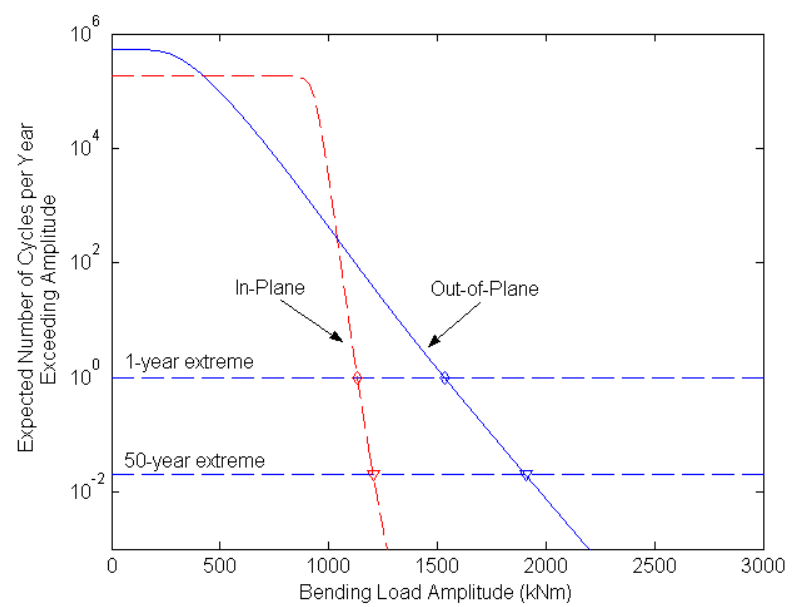

Figure 33. Expected number of cycles per year for stall-regulated turbine.

$$
n_{L T}(L)=\int_{V_{\text {in }}}^{V_{\text {out }}} \int_{0}^{\infty} P\left[L_{a}>L \mid V, \sigma\right] n(V, \sigma) f_{V, \sigma}(V, \sigma) d \sigma d V
$$

where $n_{L T}(L)$ is the long-term, unconditional expected number of cycles larger than $L$ in $10 \mathrm{~min}$, $n(V, \sigma)$ is the number of cyclic amplitudes in a 10-minute simulation above the threshold value and $f_{V, \sigma}(V, \sigma)$ is the joint probability density function of the wind conditions. Note that, because the short-term probability distributions are fit to the upper $1 \%$ of the data, the long-term cyclic distributions are only valid for extrapolating to long-return-period, large-amplitude cycles and will not necessarily reflect the probability distribution at lower amplitudes. It is also important to notice that the number of cyclic amplitudes for a fixed time duration varies with wind condition and must be included inside the integral to account for the changing sample rate.

The long-term cyclic distributions for the pitch and stall-regulated turbines are shown in Figures 32 and 33 respectively. These distributions are shown as the number of cycles per year, which is easily obtained by multiplying Equation 38 by the number of 10 -minute periods in one year $(52,596)$. The horizontal lines in each of these figures represent the 1 - and 50-year return periods. The long-term distribution curves cross these horizontal lines at the estimated extreme 1- and 50year loading amplitude values. For both turbines, the extrapolated amplitudes for the out-ofplane load are larger than the extrapolated in-plane loads. Therefore, the out-of-plane fatigue loads are likely to contribute the most damage and drive the fatigue design of the turbine. However, notice that for the stall-regulated turbine (Fig. 33), the distribution curves are higher for the in-plane loading at lower amplitude values. Depending on how fatigue damage accumulates, these lower level loads with a greater number of cycles could produce damage equivalent to or greater than the out-of-plane loading.

\section{Lifetime estimation}

Estimates of the lifetime of a turbine blade were calculated using the accumulated damage from the data below the threshold and the short-term probability fits to the amplitude data above the threshold as in the following formulas 


$$
\begin{gathered}
\text { Lifetime }=\int_{V_{\text {in }}}^{T / V_{\text {out }}^{\infty}} \int_{0}^{\infty}\left(D_{0}(V, \sigma)+D_{t h}(V, \sigma)\right) f_{V, \sigma}(V, \sigma) d \sigma d V \\
D_{0}(V, \sigma)=\sum_{i<i_{t h}} \frac{1}{N\left(L_{i}(V, \sigma)\right)} \\
D_{t h}(V, \sigma)=\int_{L_{t h}(V, \sigma)}^{\infty} \frac{n(V, \sigma) f_{L \mid V, \sigma}(L \mid V, \sigma)}{N(L)} d L \\
N(L)=\left(\frac{L}{L_{u l t}}\right)^{-m}
\end{gathered}
$$

where $D_{o}(V, \sigma)$ is the damage fraction for all cycles, $L_{i}(V, \sigma)$, that are below the threshold, $L_{t h}(V$, $\sigma)$ is the threshold at a given wind condition, $D_{t h}(V, \sigma)$ is the equivalent damage fraction of the fitted distribution above the threshold, $f_{L \mid V, \sigma}(L \mid V, \sigma)$ is the fitted conditional density function for the loading amplitude above the threshold, $n(V, \sigma)$ is the number of cycles above the threshold for a given time period $T, f_{V, \sigma}(V, \sigma)$ is the joint probability density function for the wind conditions, $N$ is the number of cycles to failure for a given load amplitude, and $m$ is the material fatigue exponent.

As mentioned above, the ultimate bending strength for each turbine blade, $L_{u l t}$, was based on a probabilistic extrapolation of extreme loads. The above formulas assume that Miner's rule of linear accumulated damage with a power law S-N curve is valid [19]. The lifetime calculations also assumed that the fatigue design was dominated by the larger out-of-plane bending loads and the in-plane loads were ignored. Load cycles arising from non-operating design situations were also neglected.

Figures 34 and 35 show the estimated lifetime for both turbines as a function of the material exponent. In both of these figures there are two curves: one that represents the lifetime estimated only from the data (above and below the threshold) and the other that replaces the data above the threshold with the fitted distribution, as described above. For most exponent values the difference between using only the data and adding the damage from the fitted distribution is insignificant. At the highest exponent values the differences between the two curves increases slightly. The increasing difference as a function of material exponent arises from more damage being done by the large amplitude cycles when the material exponent is also large. Using the fitted distribution above the threshold adds some more damage resulting from the rare large amplitudes that may be missing in the simulation data. Because the differences between the data and fit are so small, using fitted distributions to extrapolate the damage may be unnecessary. However, if less data were available for calculating damage, the differences between the predicted lifetimes for the extrapolation and the data would be larger.

Another significant result noticeable in these figures is the relative difference in fatigue lifetimes for the different material exponents. For a typical wind turbine design, a reasonable expected lifetime would be close to 30 years or longer, when considering appropriate margins for structural reliability. In these figures, lifetimes longer than 30 years are expected only for the highest values of material exponent. Reasonable lifetimes are obtained for exponent values only above 6 


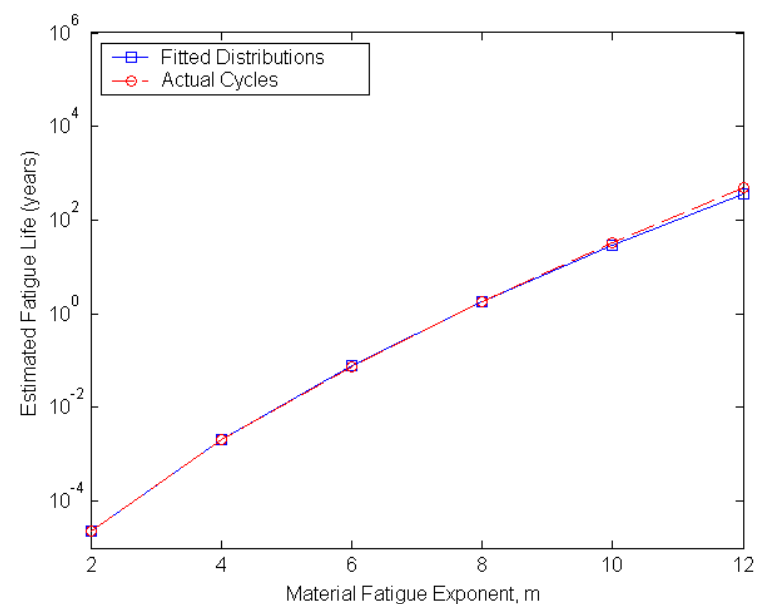

Figure 34. Estimated fatigue life for pitchregulated turbine.

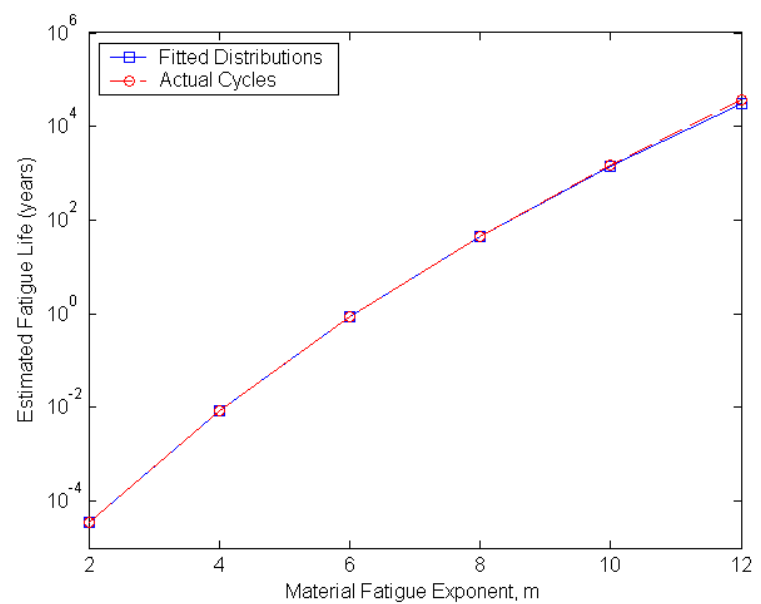

Figure 35. Estimated fatigue life for stallregulated turbine.

for the stall-regulated turbine and above 8 for the pitch-regulated turbine. For a lower material exponent of 3, the estimated lifetimes for both turbines are on the order of several hours, which is obviously not economically realistic. For these types of materials, which are typically metals (steels, etc.), fatigue considerations dominate over considerations of ultimate loading. Therefore, the ultimate strength of the turbine must be increased in order to obtain a reasonable fatigue design lifetime. This will lead to a much larger design margin for ultimate loading.

\section{Simplified model}

To reduce the number of simulations required, we saw above that the inputs to the simulations can be increased to approximate extrapolated long-term extreme values. For fatigue extrapolation, we propose that the turbulence level be assigned the same deterministic value of the 90th percentile level calculated from the original log-normal conditional distribution. This procedure results in a fairly simple equation for turbulence level given by

$$
\sigma(V)=I_{r e f}(0.8 V+b)
$$

where the values of $I_{r e f}$ and $b$ are 0.15 and 6 respectively, as stated above.

By using a deterministic turbulence level, one level of integration is eliminated from the longterm probability calculation. Hence, Equation 38 becomes

$$
n_{L T}(L)=\int_{V_{\text {in }}}^{V_{\text {out }}} P\left[L_{a}>L \mid V\right] n(V) f_{V}(V) d V
$$

and Equation 39 becomes

$$
\text { Lifetime }=\iint_{V_{\text {in }}}^{T / V_{\text {out }}}\left(D_{0}(V)+D_{t h}(V)\right) f_{V}(V) d V
$$

and similar reductions are applied to Equations 40 and 41. 


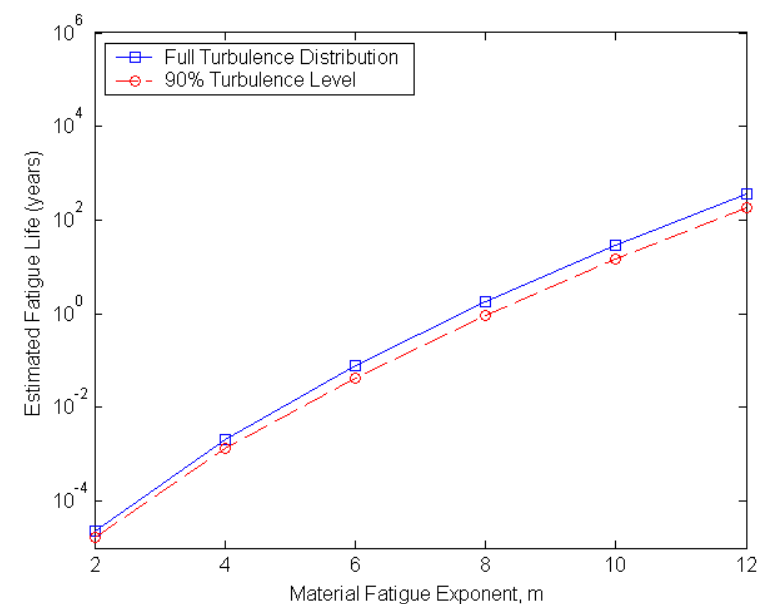

Figure 36. Estimated fatigue life for pitchregulated turbine using deterministic turbulence level.

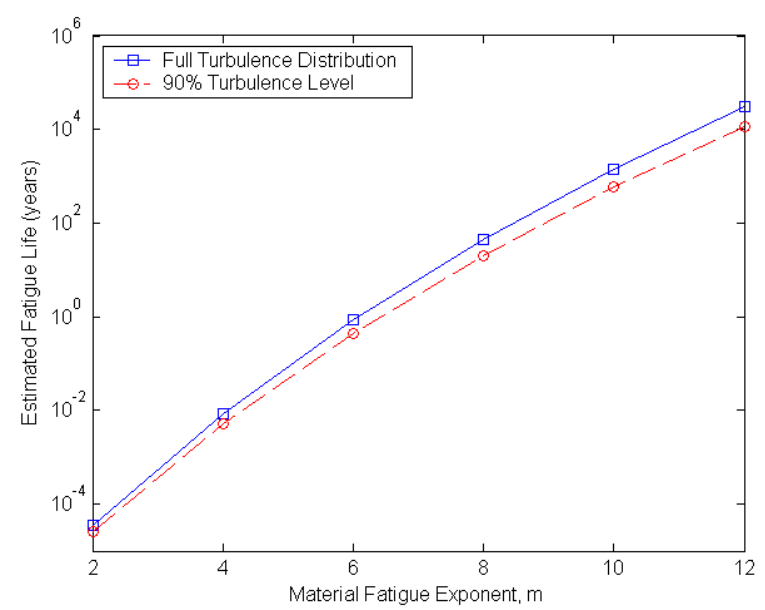

Figure 37. Estimated fatigue life for stallregulated turbine using deterministic turbulence level.

Again, nine simulations per wind condition were calculated requiring only 189 simulations instead of 4725 simulations for the full distribution method.

Figures 36 and 37 compare the estimated lifetimes using the original full distribution of wind speed and turbulence level to the lifetime estimated using the deterministic 90th percentile turbulence level at each wind speed. For both turbines, the estimated fatigue life using the deterministic turbulence level follows the same trend as lifetime calculated from the full distribution, but the lifetime from the deterministic turbulence level is lower (i.e. conservative). Because the differences in lifetimes between the two distributions are less than an order of magnitude, using the deterministic turbulence level may be advantageous. The fact that, for both turbines considered, the lifetime prediction using this simplification was conservative adds to its appeal. However, caution must be exercised in general, because it is possible that certain nonlinear loading effects leading to much higher load sensitivity to turbulence could lead to non-conservative results.

\section{One-year Simulation}

To determine their accuracy, results from the statistical load extrapolation methods were compared to direct simulations of operating loads for a 1-year period. Because the direct simulation was done with the same simulation codes (SNWind [12] and FAST [13]) to acquire data as the extrapolation methods, they both should produce nearly identical long-term distributions for one year.

To produce one-year's worth of data, both wind turbine models were simulated for an entire year in 10-minute segments. Using the joint density function of wind speed and turbulence level described by Equations 1-9, the number of 10-minute simulations per wind condition were determined by randomly sampling values from the joint probability distribution, 52,596 times (the number of 10-minute periods per year). If the value of the mean wind speed was below $5 \mathrm{~m} / \mathrm{s}$, the wind condition was eliminated, because the turbines, with startup wind speeds of approximately $5 \mathrm{~m} / \mathrm{s}$, will likely never operate at these low wind speeds. Wind speeds above the cut-out wind speeds $(25 \mathrm{~m} / \mathrm{s})$ were also eliminated to create a data set consistent with the long-term extrapolation method. Transients that occur from either startup or shut down of the turbine near the limits of its operating range were neglected. After eliminating the non-operating wind speeds, approximately 43,000 simulations remained. The numbers of simulations per wind condition are 


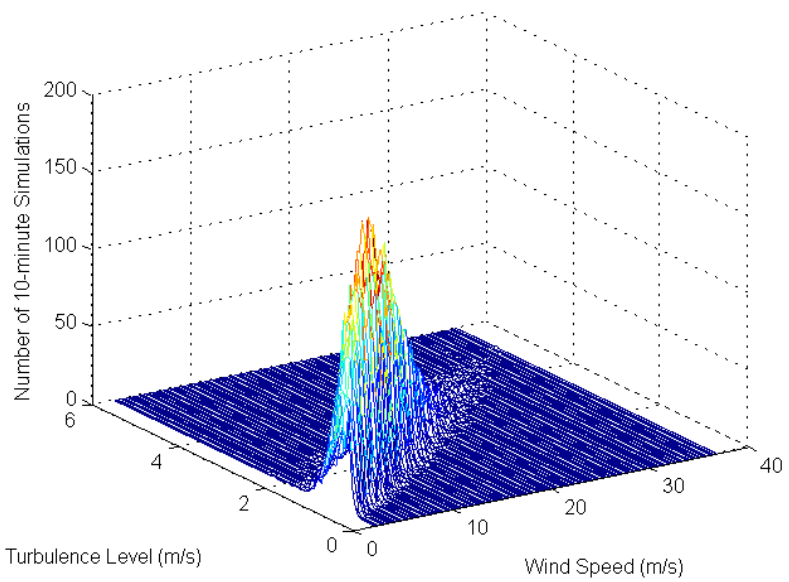

Figure 38. Total number of 10-minute simulations per wind condition in one-year

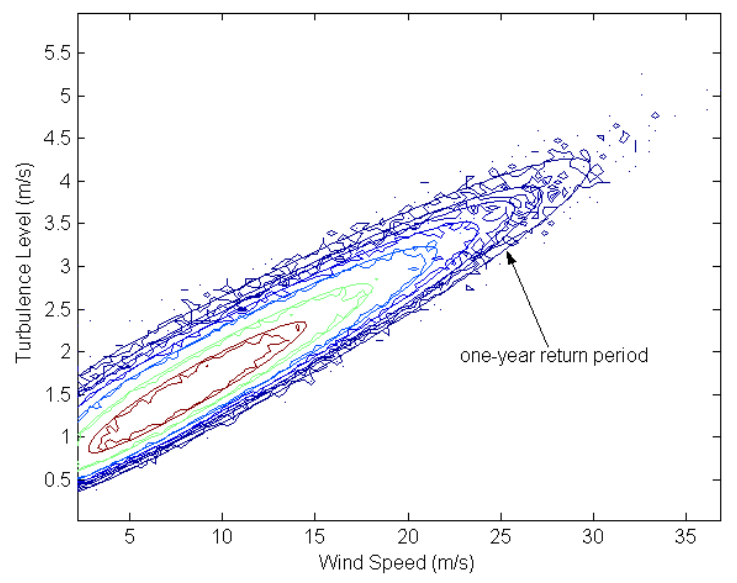

Figure 39. Contour of number of 10-minute simulations per wind condition and joint probability density function

shown graphically in Figures 38 and 39. Note that in Figure 39, the contour of joint probability density function (smooth lines) is plotted along with the contour of the actual number of cycles. This demonstrates that some simulations had wind conditions that were outside of the one-year return period, which is statistically realistic.

Because of the large number of simulations, it was essential that none of the random wind conditions were repeated. To ensure that this occurred, the final seed numbers of an SNWind simulation were used as the initial inputs into the following simulation. This process was repeated until all simulations were complete. Because the random number generator in SNWind has a return period on the order of $2 \times 10^{18}$, the stochastic component of each simulation was uncorrelated with all other simulations.

\section{Extreme Values}

Figure 40 compares the data from the direct one-year simulation to the predicted long-term distributions of extreme loads using the full integration method. In this figure, asterisks represent the data distributions from each of the three blades on the turbine. The three different data sets highlight the variability of the simulated long-term distribution. This variability is highest for the largest extreme values because of their low frequency of occurrence and hence greatest uncertainty. In the figure, we can see that the predicted distribution of the in-plane loads matches the actual distribution from the direct simulation very well. At the highest values near the one-year extreme, the predicted distribution slightly underestimates some of the data, but the errors are on the order of $5 \%$. On the other hand, the predicted distribution for the out-of-plane loads greatly overestimates the actual distribution, where the difference in predicted one-year extreme values is close to $20 \%$. This difference can be attributed to a failure of the short-term distributions to follow the trends in the tails of the data, especially near rated wind speed and higher levels of turbulence intensity. As stated above, one may have to use a different method of fitting the data distributions for pitch-regulated turbines if this error in the predicted distribution is to be reduced.

Figure 41 compares the predicted long-term distributions of extreme loads to the data from the direct one-year simulation. In this figure, predictions of both the in-plane and out-of-plane loads agree well with the actual data. Prediction of the one-year extreme out-of-plane load is slightly 


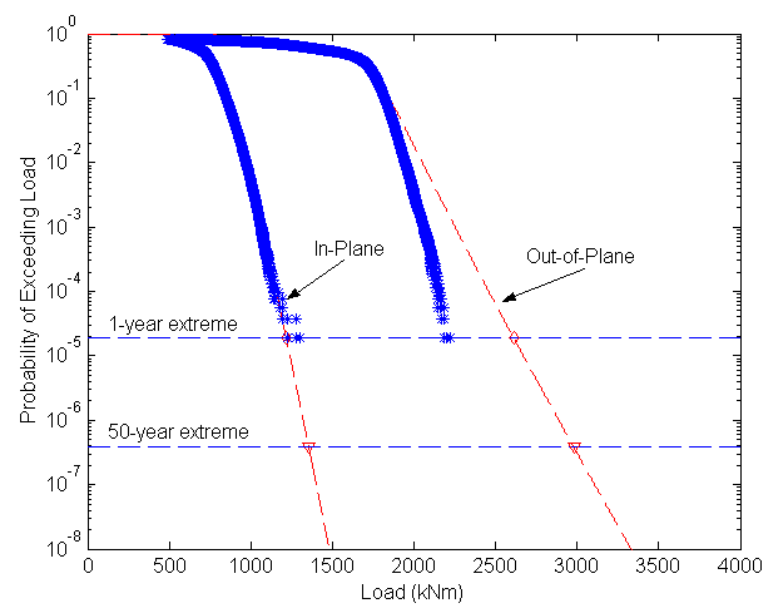

Figure 40. Predicted long-term distributions of extreme loads compared to direct simulation data for pitch-regulated turbine.

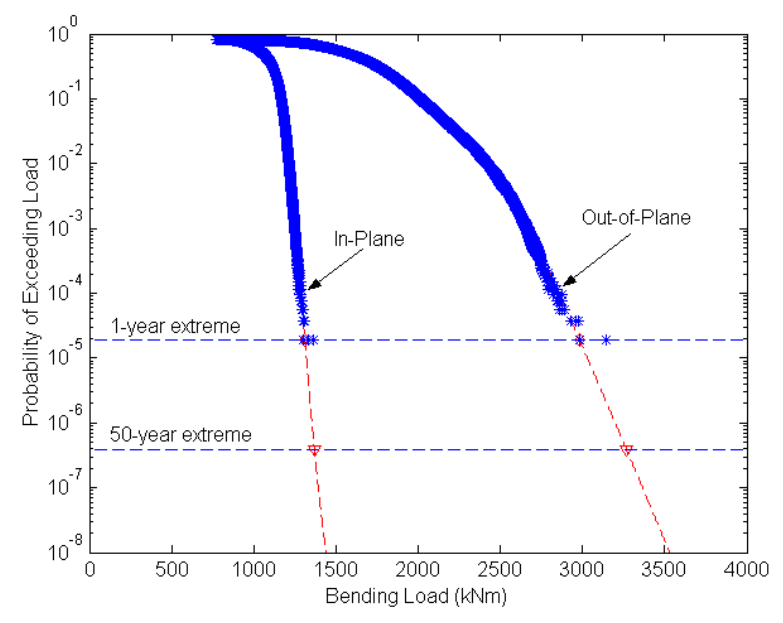

Figure 41. Predicted long-term distributions of extreme loads compared to direct simulation data for stall-regulated turbine.

less than some of the actual data, which can be attributed to the random uncertainty of the data for these extreme values.

\section{Fatigue}

Figures 42 and 43 show comparisons of the long-term distributions of fatigue cycles compared to data from the direct one-year simulation for both turbines. Again, asterisks represent the data of three different data sets, one for each blade. As with the extreme values, the predicted fatigue distributions for the in-plane loads of both turbines agreed very well with the actual data distributions. However, for the out-of-plane loads, the agreement is not as good, particularly for the pitch-regulated machine. Again, this is likely due to the inability to accurately predict the shortterm distributions at each wind condition using the method of moments.

Because the out-of-plane loads are overpredicted for both turbines, one would expect that lifetime predictions using extrapolation would estimate shorter lifetimes than actually would occur. As seen in Figures 44 and 45, this is the case, although the differences are not large. For most

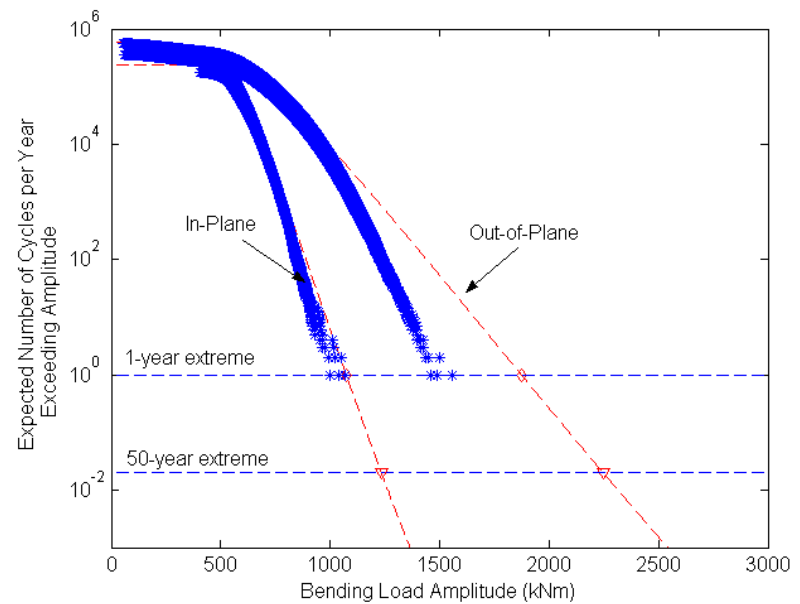

Figure 42. Predicted long-term distributions of fatigue loads compared to direct simulation data for pitch-regulated turbine.

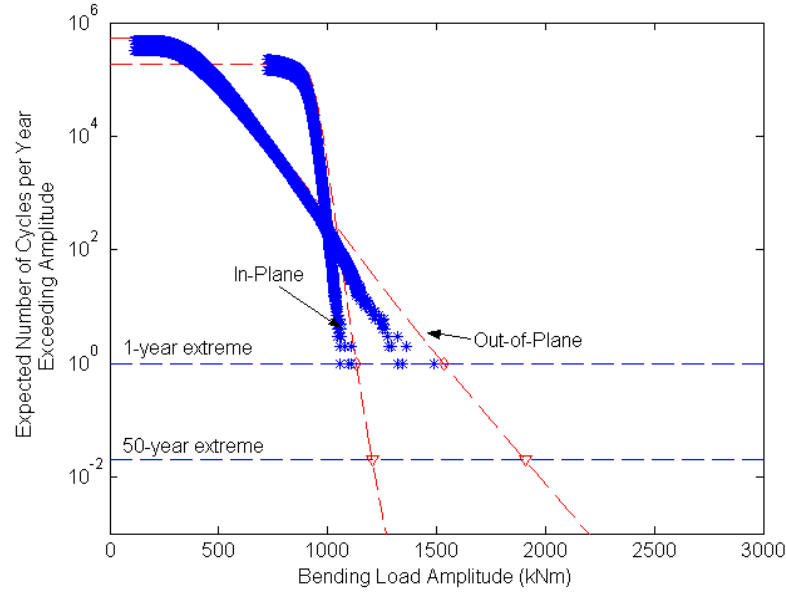

Figure 43. Predicted long-term distributions of fatigue loads compared to direct simulation data for stall-regulated turbine. 


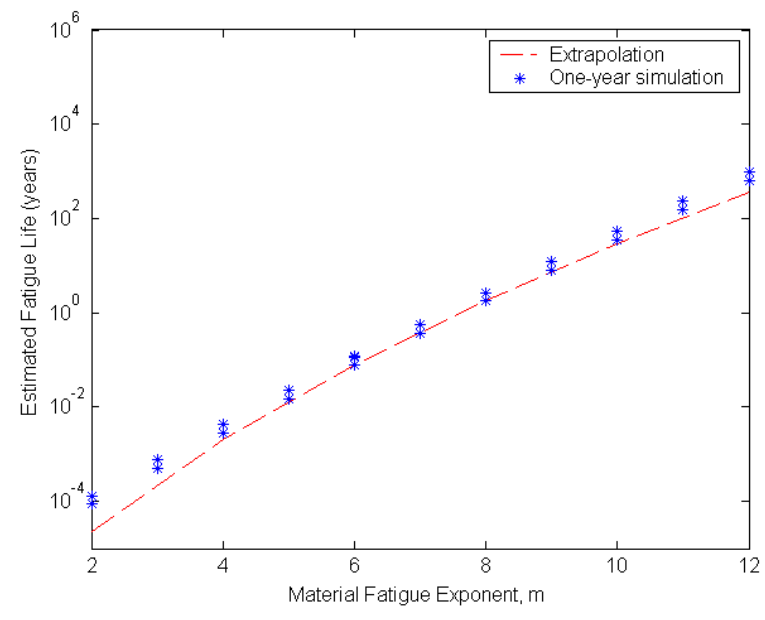

Figure 44. Predicted lifetimes compared to direct simulation data for pitch-regulated turbine.

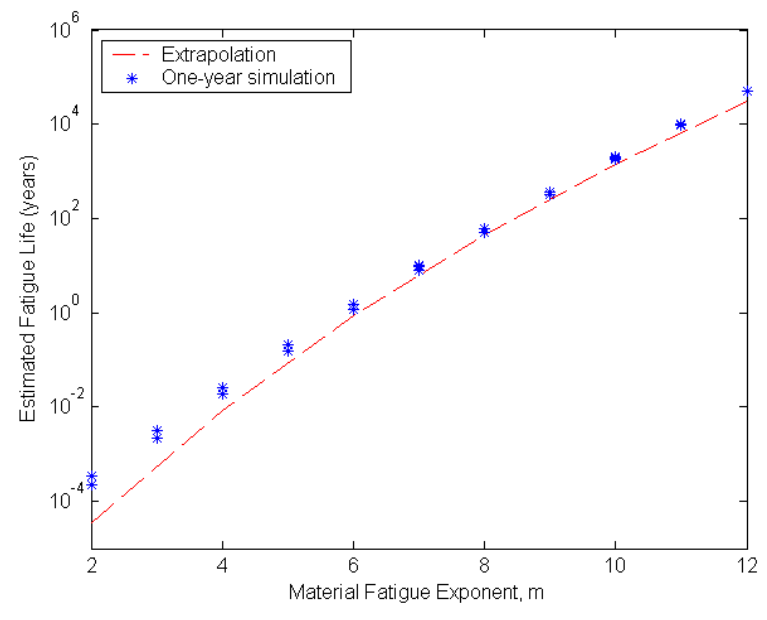

Figure 45. Predicted lifetimes compared to direct simulation data for stall-regulated turbine.

material fatigue exponents, the extrapolated lifetime is less than predicted when using one-year's worth of data. The error is greatest at the lowest values of material fatigue exponent, which represents materials like steel etc. Interestingly, for the pitch-regulated turbine, the agreement between one-year simulation data and extrapolation is very good for material fatigue exponents between 6 and 8. The implication of this is that the large difference between distributions seen in Figure 42 does not result in a large difference in predicted fatigue life for these material exponents. In other words, fatigue cycles with the largest amplitudes will not dominate the fatigue spectrum of this turbine, but lower amplitudes cycles with greater frequency will instead.

Another interesting trend seen in these figures is that the predicted lifetimes using extrapolation were within one order of magnitude or less of the one-year simulation estimate, which is often considered the error band for fatigue estimates using Miner's rule. Finally, considering the discussion of Figures 34 and 35, one can conclude that using only 4725 simulations of the turbines operating in a representative wind environment produced nearly identical fatigue life estimates to an entire year's worth of simulations.

\section{Conclusions}

We found probabilistic methods to be useful for extrapolating extreme wind turbine bending loads from a limited data set. Compared to a direct simulation of one year's worth of operation, the probabilistic methods accurately predicted the extreme loading distribution of in-plane bending loads on both a pitch and stall-regulated machine, as well as out-of-plane loads on the stall regulated turbine. The methods used overpredicted the extreme loads in the out-of-plane direction for the pitch-regulated turbine by $20 \%$ or more. This overestimation was due to the method of moments' inability to accurately model the extreme distribution of these loads near rated wind speed and at high turbulence intensities. On pitch-regulated machines, this operating regime is marked by a change in loading behavior that may require alternative probability methods to model correctly.

The choice of distribution used to model loads dramatically affected the predicted long-term extreme loads. A regular Weibull model was most conservative, followed by a Gumbel, threeparameter Weibull, and finely quadratic Weibull. 
Using a threshold for selection of the extreme values was found to improve the estimate of the long-term loads. By only selecting the largest values between successive up-crossings of the threshold, the bias introduced in the statistical moments due to the more frequent smaller local maxima is removed and the retained local maxima are less correlated. The resulting estimated distribution more closely follows the trend of the largest extreme values. An acceptable threshold value for both in-plane and out-of-plane extreme blade loads was found to be the mean value plus 1.4 times the standard deviation of the original time series for the quantity of interest.

Higher-order moments, such as the standard deviation and skewness of the extreme distribution, were found to have the greatest amount of uncertainty and also the largest influence on the extrapolated long-term loads. Some of this uncertainty was mitigated by using larger data sets of 10-minute simulations, smoothing the moments with neighboring wind conditions, and parametrically modeling the moments before calculating a fitted distribution. Using a parametric model of the data moments was also valuable for reducing the amount of data needed to predict longterm loads. The estimated loads were within $5 \%$ of those calculated with a full integration method.

A deterministic model of the turbulence level was used to simplify the calculation of the longterm probability distributions of extreme loads. A 90th percentile turbulence level was found to predict in-plane loads similar to those predicted by the full integration method and out-of-plane loads that were $5 \%$ to $10 \%$ more conservative than the full integration method. This elevated turbulence level may provide a useful simplification for designers looking for a quicker implementation of loads extrapolation techniques.

Extrapolating the turbulence level before input into the aeroelastic codes may also be a useful method for reducing the data requirements of extreme extrapolation, although a more sophisticated method, such as inverse FORM, is recommended for better accuracy.

The usefulness of probabilistic methods to predict fatigue damage was less certain. As with extreme loads, probabilistic methods produced cyclic amplitude distributions of in-plane loads very similar to data extracted from a direct one-year simulation. The same methods overpredicted the out-of-plane amplitude distribution and may be unnecessarily conservative. Using probabilistic fits to determine fatigue lifetimes produced nearly identical results to using raw rainflow cycles extracted from the time series, and therefore may be redundant. However, if a smaller data set was used with a size typical for industrial design, probabilistic fitting of the data may have produced more conservative and probably more accurate estimates of the fatigue lifetime. Using the large data set of 4725 simulations in this study produced lifetime estimates on the same order as data from an entire year's worth of simulation.

A Goodman correction was found to be useful for correcting rainflow counted amplitudes with nonzero midpoints. Inclusion of this correction increases the equivalent damage for a given amplitude and should produce more accurate results. Although, the accuracy of this correction is most likely a function of the material properties and should be studied further.

Using a threshold value of the 99th percentile of the amplitude distribution greatly increased the accuracy of the moment-based methods to follow the trend in the tail of the data. Also, using a limited data set of nine simulations was found to be statistically similar to a much larger data set, which inherently has statistical moments with lower uncertainty.

Out-of-plane bending loads were determined to produce larger amplitude cycles than the in-plane loads. Because of this, these loads where assumed to dominate the fatigue loading of the turbine designs. 
Fatigue lifetime varied greatly with material fatigue exponent values. For low exponent valued materials, such as steel, it was found that the fatigue component of the design dominates relative to the ultimate loading component.

A simplified probabilistic method using an artificially elevated turbulence intensity was found to produce slightly conservative lifetime estimates relative to the full distribution method. Because of its reduced data needs, this method may provide a useful tool for designing turbines similar to the ones in this study.

\section{Future work}

Continuation of this work should address the failure of the method of moments to accurately predict short-term distributions of the pitch-regulated wind turbine under certain operating conditions. Alternative methods for probabilistic fitting should be examined, such as maximum likelihood or regression methods. The extrapolation methods could also be applied to experimental test data gathered from operating wind turbines. Other areas of research that would be of interest and are currently being studied by other researchers, are environmental extrapolation (e.g. inverse FORM) methods that greatly reduce the number of simulations required for design purposes. Finally, the authors recommend further examination of fatigue extrapolation. For example, as was alluded to in this study, extrapolation was not required to estimate the fatigue lifetime of the turbine, if a large data set was available. However, if the data set used for extrapolation was smaller, the extrapolation technique may have proven useful. The limit of data set size at which extrapolation becomes useful would be of interest.

\section{Acknowledgements}

The authors would like to thank LeRoy Fitzwater and Steve Winterstein of Stanford University for guidance and use of the FITS code, as well as a peak finding algorithm. Also, discussions with Paul Veers of Sandia National Laboratories were valuable in implementing alternative methods for loads extrapolation.

\section{References}

[1] IEC/TC88, 61400-1 ed. 2, Wind turbine generator systems-part 1: Safety Requirements, International Electrotechnical Commission (IEC), 1998.

[2] Veers, P. S., Winterstein, S. R., "Application of measured loads to wind turbine fatigue and reliability analysis," Proc., 1997 ASME Wind Energy Symposium, 35th AIAA Aero. Sci. Mtg., pp. 160-169, January, 1997.

[3] Ronold, K. O., Wedel-Heinen, J. W., Christensen, C. J., "Reliability-based fatigue design of wind-turbine rotor blades," Engineering Structures, 21, pp. 1101-1114, 1999.

[4] Ronold, K. O., Larsen, G. C., "Reliability-based design of wind-turbine rotor blades against failure in ultimate loading," Engineering Structures, 22, pp. 565-574, 2000.

[5] Madsen, P. H., Pierce, K., Buhl, M., "Predicting ultimate loads for wind turbine design," Proc., 1999 ASME Wind Energy Symposium, 37th AIAA Aero. Sci. Mtg., pp. 355-364, January, 1999.

[6] Thomsen, K. The statistical variation of wind turbine fatigue loads. Risø-R-1063 (EN), Roskilde, Denmark: Risø National Laboratory ,1998. 
[7] Fitzwater, L. M., Winterstein, S. R., "Predicting design wind turbine loads from limited data: comparing random process and random peak models," Proc., 2001 ASME Wind Energy Symposium, 39th AIAA Aero. Sci. Mtg., pp. 355-364, January, 2001.

[8] Manuel, L., Veers, P. S., Winterstein, S. R., "Parametric models for estimating wind turbine fatigue loads for design," Proc., 2001 ASME Wind Energy Symposium, 39th AIAA Aero. Sci. Mtg., pp. 276-287, January, 2001.

[9] Fitzwater, L. M., Winterstein, S. R., Cornell, C. A., "Predicting the long term distribution of extreme loads from limited duration data: comparing full integration and approximate methods," Proc., 2002 ASME Wind Energy Symposium, 40th AIAA Aero. Sci. Mtg., pp. 267-277, January, 2002.

[10] Moriarty, P. J., Holley, W. E., Butterfield, S., "Effect of turbulence variation on extreme loads prediction for wind turbines," Proc., 2002 ASME Wind Energy Symposium, 40th AIAA Aero. Sci. Mtg., pp. 278-287, January, 2002.

[11] Larsen, D.C., Ronold, K. O., Jørgensen, H. E., Argyriadis, K., de Boer, J., Ultimate loading of wind turbines, Risø-R-1111 (EN), Risø National Laboratory, Roskilde, Denmark, April, 1999.

[12] Kelley, N. D., "Full Vector (3-D) Simulation in Natural and Wind Farm Environments Using an Expanded Version of the SNLWIND (Veers) Turbulence Code," Wind Energy 1993, S.M. Hock (ed.), SED-Vol.14, ASME, 1993.

[13] Buhl, M. L., Jonkman, J. M., Wright, A. D., Wilson, R. E., Walker, S. N., Heh, P., FAST User's Guide, NREL/EL-500-29798, National Renewable Energy Laboratory, Golden, CO, 2002.

[14] Dowling, N.E. Fatigue Failure Predictions for Complicated Stress-strain Histories. Journal of Materials Vol.7 (No.1): 71-87, 1972.

[15] Manuel, L., Kasef, T., Winterstein, S. R., Moment-based probability modeling and extreme response estimation - The FITS routine Version 1.2, SAND99-2985, Sandia National Laboratories, Albequerque, NM, Nov., 1999.

[16] Abramowitz, M. and Stegun, I. A., Handbook of Mathematical Functions, 10th edition, Dover, 1974.

[17] Fitzwater, L. M., Cornell, C. A., Veers, P. S., "Using environmental contours to predict extreme events on wind turbines," Proc., 2003 ASME Wind Energy Symposium, 41st AIAA Aero. Sci. Mtg., pp. 244-258, January, 2003.

[18] Saranyasoontorn, K., Manuel, L., "A comparison of wind turbine design loads in different environments using inverse reliability," Proc., 2004 ASME Wind Energy Symposium, 42nd AIAA Aero. Sci. Mtg., pp. 151-161, January, 2004.

[19] Sutherland, H. J., On the fatigue analysis of wind turbines, SAND99-0089, Albuquerque, NM, Sandia National Laboratories, 1999. 


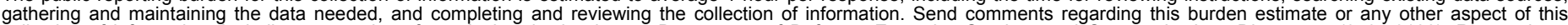

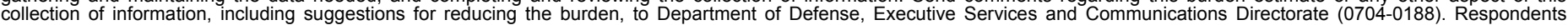

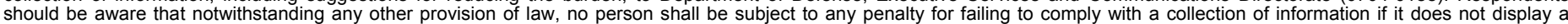

currently alid OMB control number.

PLEASE DO NOT RETURN YOUR FORM TO THE ABOVE ORGANIZATION.

\begin{tabular}{l|l|l|}
\hline 1. REPORT DATE $(D D-M M-Y Y Y Y)$ & 2. REPORT TYPE & 3. DATES COVERED (FrOm - TO)
\end{tabular}

November 2004

Technical Report

4. TITLE AND SUBTITLE

Extrapolation of Extreme and Fatigue Loads Using Probabilistic Methods 5a. CONTRACT NUMBER

DE-AC36-99-G010337

5b. GRANT NUMBER

5c. PROGRAM ELEMENT NUMBER

5d. PROJECT NUMBER

NREL/TP-500-34421

5e. TASK NUMBER

WER3.2040

5f. WORK UNIT NUMBER
7. PERFORMING ORGANIZATION NAME(S) AND ADDRESS(ES)

National Renewable Energy Laboratory

1617 Cole Blvd.

Golden, CO 80401-3393
8. PERFORMING ORGANIZATION REPORT NUMBER

NREL/TP-500-34421

9. SPONSORING/MONITORING AGENCY NAME(S) AND ADDRESS(ES)

10. SPONSOR/MONITOR'S ACRONYM(S) NREL

11. SPONSORING/MONITORING AGENCY REPORT NUMBER

12. DISTRIBUTION AVAILABILITY STATEMENT

National Technical Information Service

U.S. Department of Commerce

5285 Port Royal Road

Springfield, VA 22161

13. SUPPLEMENTARY NOTES

14. ABSTRACT (Maximum 200 Words)

Probabilistic methods were used to determine the long-term extreme and fatigue loads of two different $1.5 \mathrm{MW}$ wind turbine designs. Over the past decade, probabilistic methods for wind turbine design have acquired increasing levels of interest in bothe the research and industrial community. This interest has largely been driven by the desire to replace outdated empirical-based design models with more physically relevant models that can be accurately applied to the next generation of wind turbine designs. This study further extends previous works through further examination of moment-based probability methods, as well as the effects of using a joint distribution of mean wind speed and turbulence level, and the statistical uncertainty of moment-based methods, introducing refinements such as compensating for non-zero cyclic midpoints in fatigue calculations, application of a threshold technique and full integration of all probable operating conditions.

15. SUBJECT TERMS

probabilistic methods; fatigue loads; wind energy; wind turbine;

\begin{tabular}{|c|c|c|c|c|}
\hline 16. SECURIT & CLASSIFICATI & N OF: & 17. LIMITATION & 18. NUMBER \\
\hline $\begin{array}{l}\text { a. REPORT } \\
\text { Unclassified }\end{array}$ & $\begin{array}{l}\text { b. ABSTRACT } \\
\text { Unclassified }\end{array}$ & $\begin{array}{l}\text { c. THIS PAGE } \\
\text { Unclassified }\end{array}$ & $\begin{array}{c}\text { OF ABSTRACT } \\
\text { UL }\end{array}$ & \\
\hline
\end{tabular}

19a. NAME OF RESPONSIBLE PERSON

19b. TELEPONE NUMBER (Include area code) 